ORNL/TM-2019/127156

CRADA/NFE-16-06364

Development of a Sustainable Green Chemistry Platform for Production of Acetone and Downstream Drop-in Fuel and Commodity Products Directly From Biomass Syngas Via a Novel Energy Conserving Route in Engineered Acetogenic Bacteria

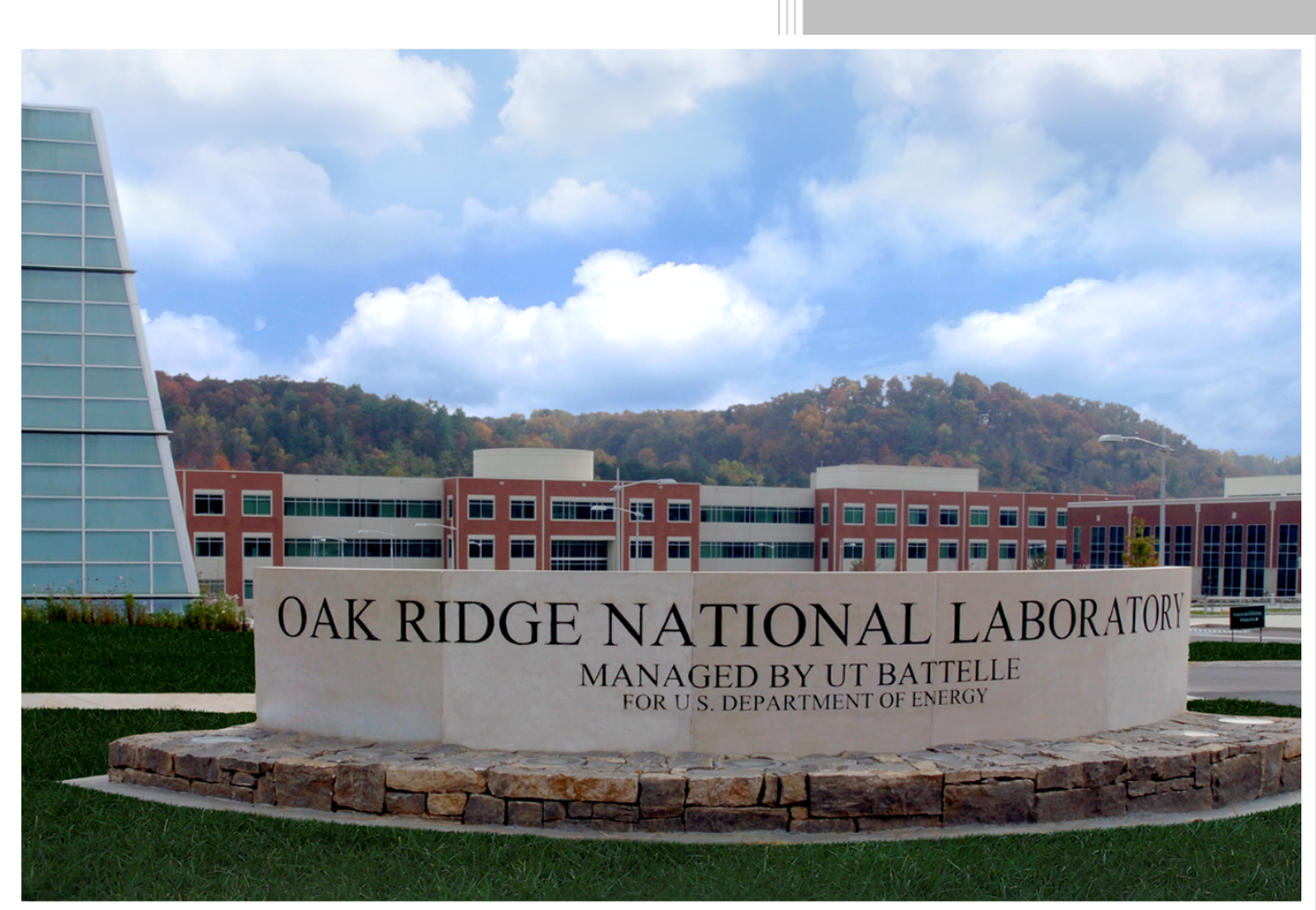

Approved for Public Release.

Distribution is unlimited. CRADA FINAL REPORT NFE-16-06364 


\section{DOCUMENT AVAILABILITY}

Reports produced after January 1, 1996, are generally available free via US Department of Energy (DOE) SciTech Connect.

Website http://www.osti.gov/scitech/

Reports produced before January 1, 1996, may be purchased by members of the public from the following source:

National Technical Information Service

5285 Port Royal Road

Springfield, VA 22161

Telephone 703-605-6000 (1-800-553-6847)

TDD 703-487-4639

Fax 703-605-6900

E-mail info@ntis.gov

Website http://www.ntis.gov/help/ordermethods.aspx

Reports are available to DOE employees, DOE contractors, Energy Technology Data Exchange representatives, and International Nuclear Information System representatives from the following source:

Office of Scientific and Technical Information

PO Box 62

Oak Ridge, TN 37831

Telephone 865-576-8401

Fax 865-576-5728

E-mail reports@osti.gov

Website http://www.osti.gov/contact.html

This report was prepared as an account of work sponsored by an agency of the United States Government. Neither the United States Government nor any agency thereof, nor any of their employees, makes any warranty, express or implied, or assumes any legal liability or responsibility for the accuracy, completeness, or usefulness of any information, apparatus, product, or process disclosed, or represents that its use would not infringe privately owned rights. Reference herein to any specific commercial product, process, or service by trade name, trademark, manufacturer, or otherwise, does not necessarily constitute or imply its endorsement, recommendation, or favoring by the United States Government or any agency thereof. The views and opinions of authors expressed herein do not necessarily state or reflect those of the United States Government or any agency thereof. 
ORNL/TM-2019/127156

CRADA/NFE-16-06364

Biosciences Division

\title{
DEVELOPMENT OF A SUSTAINABLE GREEN CHEMISTRY PLATFORM FOR PRODUCTION OF ACETONE AND DOWNSTREAM DROP-IN FUEL AND COMMODITY PRODUCTS DIRECTLY FROM BIOMASS SYNGAS VIA A NOVEL ENERGY CONSERVING ROUTE IN ENGINEERED ACETOGENIC BACTERIA
}

\author{
Author(s) \\ Timothy J. Tschaplinski (ORNL Lead PI) \\ Payal Charania, Nancy L. Engle, Richard J. Giannone, Robert L. Hettich, Dawn Klingeman, \\ Suresh Poudel, Zamin Yang \\ Oak Ridge National Laboratory
}

Sean D. Simpson (Project PI and LanzaTech, Inc. Lead PI)

Tanus Abdalla, Steve D. Brown, Christina Canter, Robert Conrado, James Daniell, Asela Dassanayake, Allan Gao, Rasmus O. Jensen, Michael Köepke, Ching Leang, FungMin (Eric) Liew, Shilpa Nagaraju, Robert Nogle, Ryan C. Tappel, Loan Tran

LanzaTech, Inc.

Date Published:

June 2019

Prepared by

OAK RIDGE NATIONAL LABORATORY

Oak Ridge, Tennessee 37831-6283

managed by

UT-BATTELLE, LLC

for the

US DEPARTMENT OF ENERGY

under contract DE-AC05-00OR22725

June 5, 2019

Approved for Public Release 


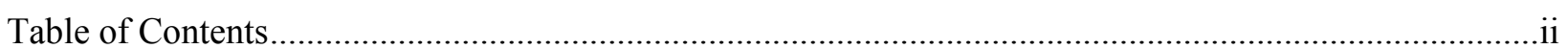

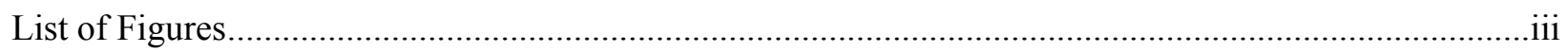

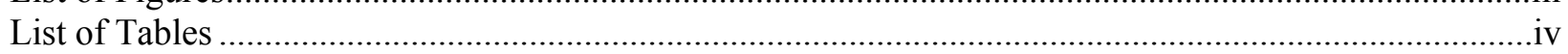

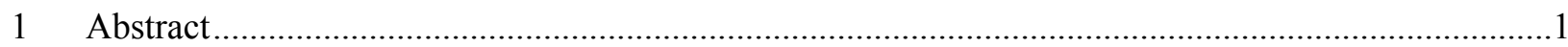

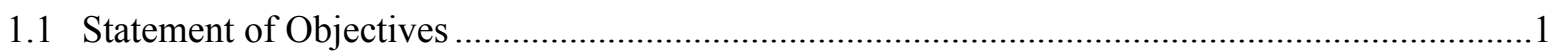

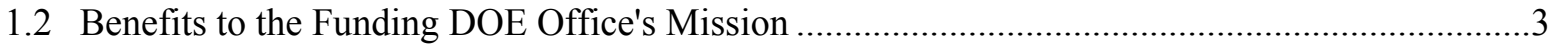

2 Technical Discussion of Work Performed by All Parties..............................................................

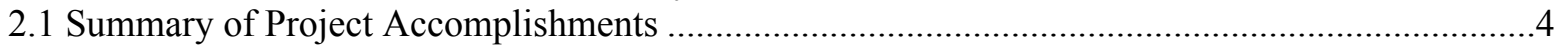

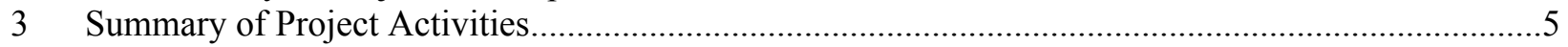

3.1 Genome Scale Model (GEM), strain engineering and fermentation optimization .....................6

3.1.1 GEM prediction of gene targets for improved acetone production ............................... 6

3.1.2 Chassis strain construction, fermentation and feedback to GEMs ................................

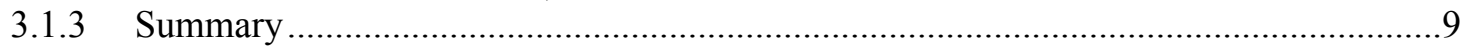

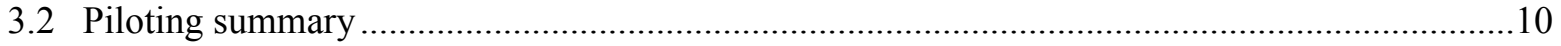

3.3 Identification of improved enzymes by genome mining of industrial strain collection ..............11

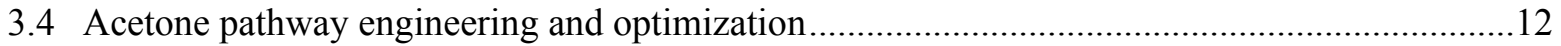

3.4.1 Combinatorial analysis of acetone pathway variants and iterative pathway

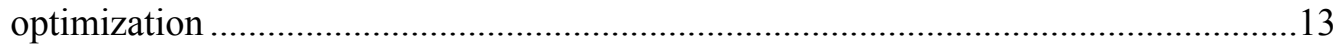

3.4.2 Platform established to screen and select Ptb-Buk variants in vitro and in vivo ..........15

3.5 Systems Approach to identify bottleneck in current fermentations .........................................16

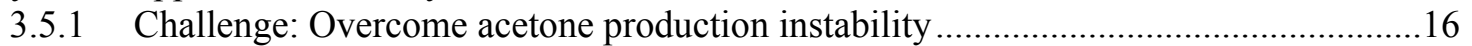

3.5.2 Understanding cellular response to acetone stress ................................................... 18

3.5.3 Development of mass-spectrometry-based approach to characterize energy

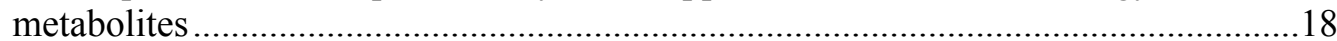

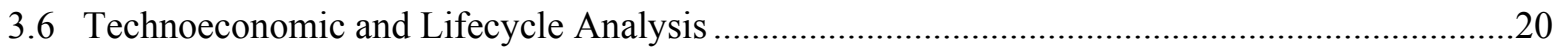

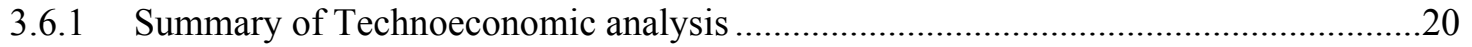

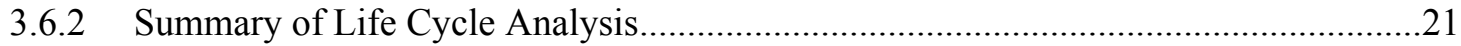

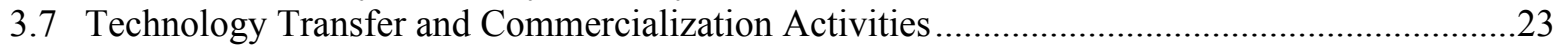

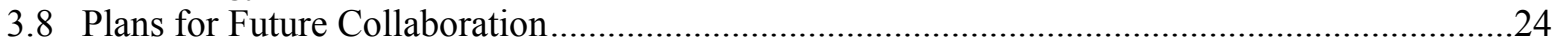

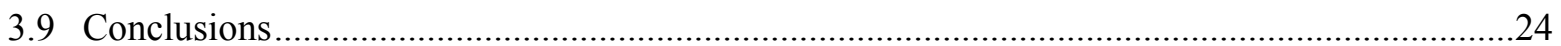

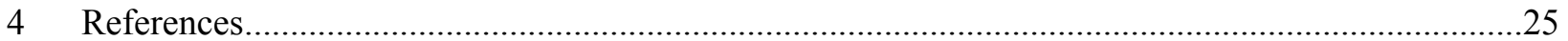




\section{List of Figures}

Figure 1. Acetone as platform chemical for fuels and bio-products...................................................2

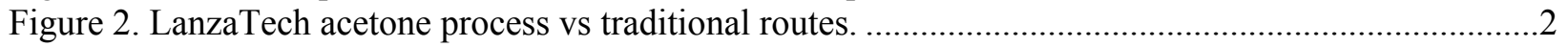

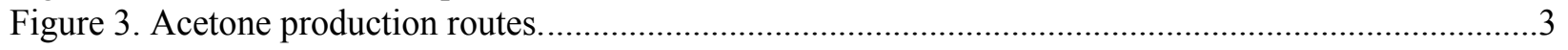

Figure 4. Approach to project goals........................................................................................

Figure 5. Production envelope showing the acetone yield/growth rate landscape for ATP-generating

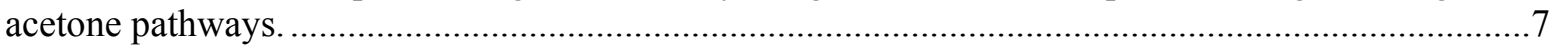

Figure 6. Good correlation between 2 L CSTR and 80 L Pilot runs .....................................................10

Figure 7. Phylogenetic trees for each gene of interest showing the relationship between the

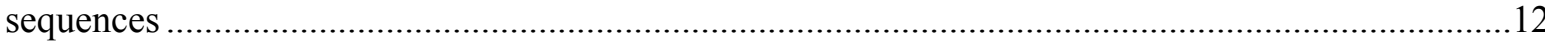

Figure 8. Distribution of end-point acetone titer by acetone combinatorial strains................................14

Figure 9. Using luminescence to screen for ATP production via Ptb-Buk reaction..................................15

Figure 10. Percent change in selectivity over $C$. beijerinckii Ptb-Buk. ..................................................15

Figure 11. Acetone production decreased over time in correlation with gene/protein expression

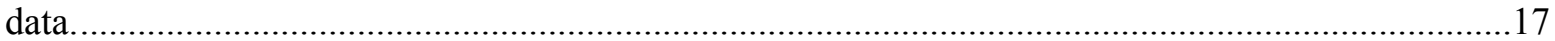

Figure 12. Minimal inhibitory concentration of acetone on cell growth ................................................18

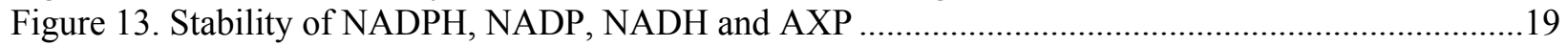

Figure 14. Technoeconomic Tradeoffs-Variation of Single Parameters. ................................................20

Figure 15. Decrease in Cash Cost of Production over the course of the project. .....................................20

Figure 16. LanzaTech process provides GHG emissions savings over traditional petrochemical

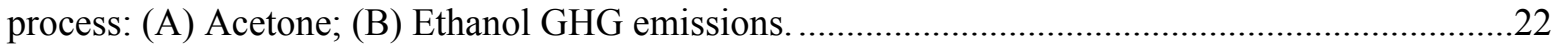


Table 1. Summary of constructed strains tested in CSTRs.

.10

Table 2. Summary of acetone pathway gene identified and mined from the first 200 completed genomes of the industrial ABE fermentation strain collection...................................................12

Table 3. Acetone pathway genes included in the targeted acetone combinatorial library ..........................13 


\section{Abstract}

LanzaTech and Oak Ridge National Laboratory have developed and scaled up a process for sustainable production of acetone and downstream drop-in fuel and commodity products directly from biomass syngas via a novel energy conserving route in engineered acetogenic bacteria. This process offers a safer and more environmentally-friendly production method for acetone production than the current phenol-dependent method, and the product has significantly lower GHG emissions. The developed process offers a cost competitive route to Acetone and enables biofuels at or below DOE's $\$ 3$ gge target. In addition, it also provides an attractive biological alternative to traditional sugar-based $\mathrm{ABE}$ fermentation, by enabling utilization of non-food biomass resources as fermentation feedstocks.

Challenges overcome:

- $\quad$ By-products formation: Byproduct 2,3-butandiol and 3-hydroxybutyrate reduce yield and stability. Addressed by elimination of both pathways in our chassis acetone production strain.

- $\quad$ Cost competitiveness: Addressed by developing an integrated acetone strain and eliminating by-products to increase yield and stability. Optimized co-selectivity and coproductivity of acetone and ethanol instead of titer, based on technoeconomic analysis.

- Limited enzyme variety: Addressed by genome mining of over 300 industrial strains in LanzaTech collection, identifying unique enzyme sequences, and refactoring these unique enzyme variants through LanzaTech's engineering platform. Resulted in over 10x improvement in acetone production from gas.

- Continuous process (stability): Identified bottleneck through detailed omics studies, addressed by integration of the acetone pathway on the chromosome.

- $\quad$ Novel process to scale up: Addressed by demonstrating stable acetone production in a 80L pilot reactor, with acetone productivity and selectivity comparable to those observed under the same conditions in 2-L reactors.

We have demonstrated stable acetone production for over 7 days at commercial target rate and selectivity in 2 -L reactors and have piloted the process.

\subsection{Statement of Objectives}

LanzaTech, Inc. (LanzaTech) partnered with Oak Ridge National Laboratory (ORNL) with the objective to develop and scale up a new process for sustainable production of acetone directly from biomass syngas using engineered acetogenic bacteria while meeting the price, quality and environmental requirements for a variety of industrial uses.

Acetone is an important industrial solvent with a global market size of 6 million tons valued at $\$ 7$ billion per annum. It is also a direct precursor of such valuable downstream products as direct drop-in fuels, fuel additives, polymers and important chemical building blocks through chemical, fermentative and even photocatalytic routes ${ }^{1-3}$ (Figure 1). 


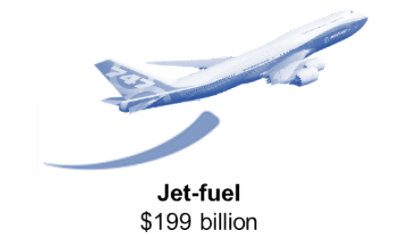

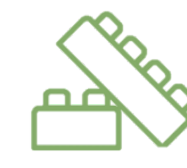

Propylene
$\$ 98$ billion

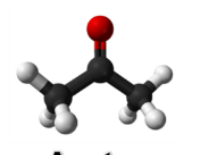

Acetone

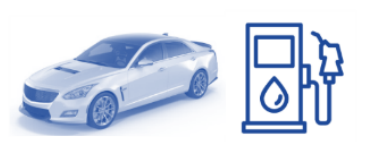

Isooctane $\$ 0.6$ billion (only US market)

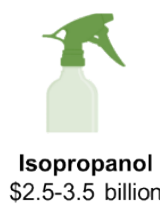

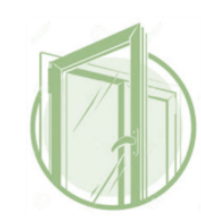

Polymethyl methacrylate (PMMA)
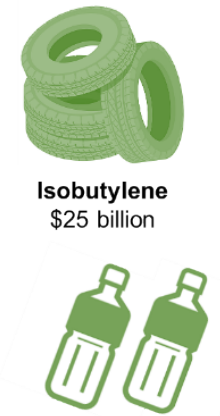

Bisphenol A (BPA) $\$ 17.7$ billion

Figure 1. Acetone as platform chemical for fuels and bio-products.

The current industrial acetone production process (the cumene process) is directly linked to petrochemical phenol production, which has significant impacts on market as well as environment ${ }^{4}$. Fermentative production of acetone (Acetone-Butanol-Ethanol or $\mathrm{ABE}$ fermentation) using sugar or molasses was carried out at industrial scale during the first half of the $20^{\text {th }}$ century, but declined thereafter due to cheap oil prices and comparatively high substrate costs (except in politically isolated countries) ${ }^{5,6}$. This project developed a green, economical process for acetone production that is decoupled from phenol production through the use of gas fermentation. The process will be safer and provide significant greenhouse gas (GHG) savings compared to acetone derived from fossil feedstocks (Figure 2).
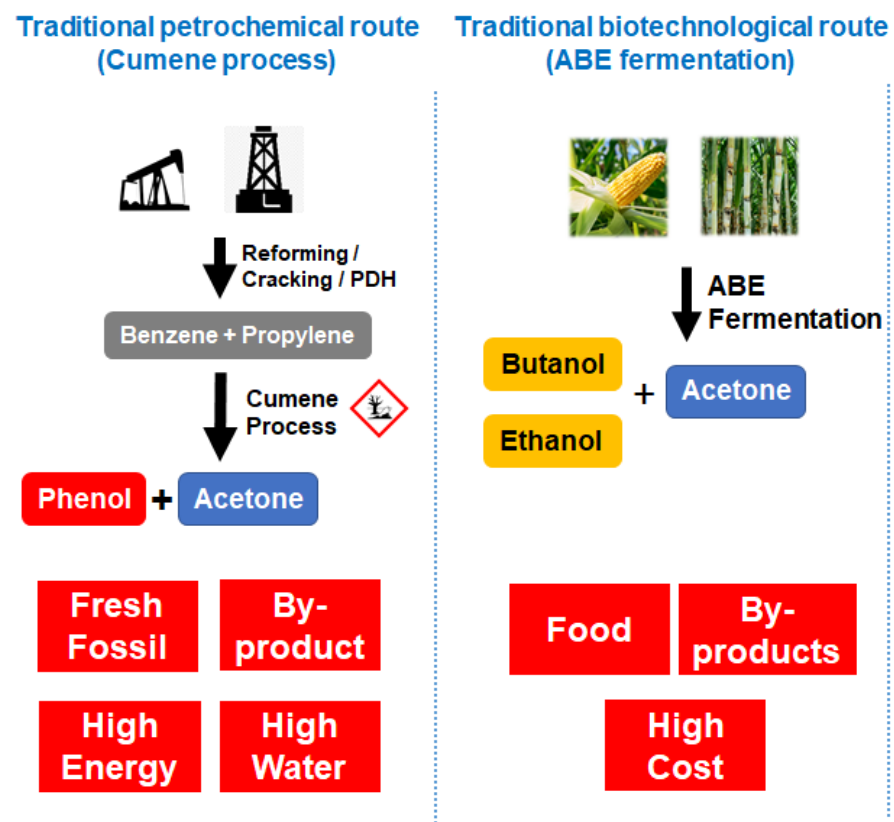

Proposed route (Gas fermentation)

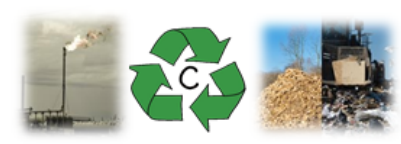

Gas

Fermentation

Ethanol $)+$ Acetone

Waste and Reduced

Residues

GHGs

Reduced

Water

Figure 2. LanzaTech acetone process vs traditional routes. 
The LanzaTech acetone process uses biomass derived syngas as feedstock and converts carbon monoxide $(\mathrm{CO})$, carbon dioxide $\left(\mathrm{CO}_{2}\right)$ and hydrogen $\left(\mathrm{H}_{2}\right)$ to acetone through implementing either the traditional acetone pathway from ABE fermentation organisms or a novel synthetic energy-conserving route into a gas-fermenting, acetogenic Clostridium bacteria via genetic engineering (Figure 3). Guided by a multi-Omics approach, a continuous production process was established, and the process was scaled up to pilot scale. The development was guided by technoeconomic (TEA) and life cycle (LCA) analyses.

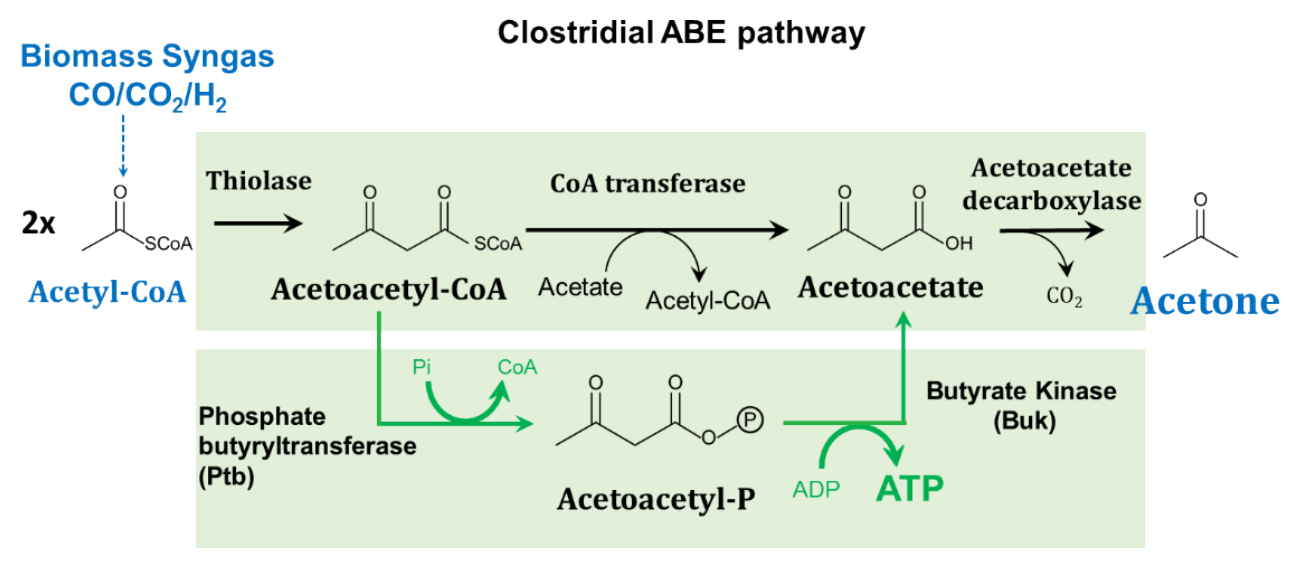

Novel synthetic pathway

Figure 3. Acetone production routes.

\subsection{Benefits to the Funding DOE Office's Mission}

The project successfully demonstrated a new stand-alone route to advanced hydrocarbon fuels and bioproducts from biomass residues with an improved safety and environmental footprint, enabling cost-competitive production of acetone as a fuel intermediate or co-product.

\section{Relevance to BETO Mission:}

- The process contributes to the Conversion R\&D goal of a commercially viable technology to convert biomass to finished liquid transportation fuels via a biological route

- Coproduction of acetone and hydrocarbon fuels will enable production of biofuels at or below BETO's target of \$3/gge target with a $180 \%$ GHG reduction over the cumene process for acetone.

- Demonstration of continuous acetone gas fermentation processes.

\section{Industry Relevance:}

- $\quad$ The acetone market value is heavily dependent on crude oil prices $(\sim \$ 3 \mathrm{~B}$ in $2015, \sim \$ 6 \mathrm{~B}$ in 2014). Production of acetone from biomass and residues will reduce price volatility and environmental impact. 
- This project offers a sustainable, cost-effective alternative to meet the needs of end users in the chemical and consumer products industries seeking to "green" their supply chains.

\section{Technical Discussion of Work Performed by All Parties}

\subsection{Summary of Project Accomplishments}

The LanzaTech-led team successfully demonstrated that biomass syngas fermentation for acetone is an economically viable, sustainable and environmental-friendly alternative to the conventional petrochemical production route, where acetone is a byproduct of phenol production using the cumene process (Figure 2). A robust microbial catalyst was developed in this project which consumes $\mathrm{CO}, \mathrm{CO}_{2}$ and $\mathrm{H}_{2}$ and produces acetone with high selectivity and productivity in a continuous, scalable process. At the end of the project, the LanzaTech acetone process was piloted and a carbon life cycle analysis (LCA) demonstrates that it offers $\geq 180 \%$ GHG emissions savings over the conventional process.

Development of a stable acetone production chassis strain. At the heart of the LanzaTech process is the robust microbial catalyst which converts $\mathrm{CO}$ and $\mathrm{H}_{2}$ to ethanol natively. It is therefore paramount to develop a chassis strain that consumes $\mathrm{CO}, \mathrm{CO}_{2}$ and $\mathrm{H}_{2}$ and continuously produces acetone and ethanol at a commercially viable rate and selectivity. Based on GEM predictions and fermentation data, several rounds of iterative strain optimizations were carried out and a total of nine new chassis strains with multiple modifications were generated. Our current best production strain is stable and co-produces acetone and ethanol at commercially viable productivities and selectivities, with all major by-products eliminated. The elimination of fermentation by-products is critical to the economics of the process as it eliminates the need for more intensive downstream separation processes.

Scale-up for the LanzaTech acetone process. Not only has LanzaTech demonstrated that the current best strain produces acetone and ethanol at a commercially viable rate and selectivity in a 2L CSTR, LanzaTech has also demonstrated acetone and ethanol co-production in a scalable 80L pilot reactor over a 4-day period. The acetone and ethanol production rate and selectivity were comparable to those achieved in a 2L CSTR under similar fermentation conditions.

Mined $>200$ genome sequences of the largest industrial ABE fermentation strain collection. In a separate effort, the JGI together with LanzaTech and ORNL, completed genome sequences for over 200 ABE fermenting industrial strains. The traditional acetone production pathway genes as well as the synthetic pathway genes were identified, and unique protein sequences were selected for combinatorial library construction which resulted in improved acetone production strains.

Largest combinatorial library for acetone pathway in acetogen. Using unique acetone pathway proteins identified through genome sequencing, a combinatorial library of over 250 combinations was constructed. In the process, we developed methods for semi-high throughput screening, with an improved understanding of the correlation between bottle growth and CSTR results. The best acetone pathway combinations were established which resulted in up to $10 \mathrm{x}$ increases in acetone production over various control pathways using genes from the industrial ABE fermentation strain collection. 
Screening method established for the synthetic pathway Ptb-Buk. A method was developed which took advantage of the ATP generated during the enzymatic reactions and coupled that ATP generation with luminescence from a firefly luciferase. Various Ptb-Buk pairs were identified from public databases (NCBI) and from the industrial ABE fermentation strain collection. Of the screened pairs, five were found to potentially have at least a $50 \%$ increase in specificity towards acetoacetyl-CoA (precursor for acetone production) over acetyl-CoA (acetate precursor) compared to a base Ptb-Buk pair.

Multi-omics studies to understand acetone production instability and strain physiology. Multiple omics platforms (transcriptomics, proteomics, metabolomics) were employed throughout the project to understand acetone production instability, strain instability, acetone stress, and energy metabolite profiles during acetone production. Based on the results derived from these studies, we constructed a commercial ready strain with the acetone pathway integrated and by-products synthesis eliminated.

Technoeconomics and Life Cycle. The technoeconomic analyses (TEA) were carried out to inform the overall strategic direction of the project, the economic and life cycle impacts and the post project commercialization strategy. The TEA considered five cases from the project including data from the $80 \mathrm{~L}$ scalable reactor run. At the end of the project, the cash cost of production (CCOP) was reduced by over $60 \%$ compared to the start of the project, with the final CCOP within $6 \%$ of our commercial target.

LanzaTech developed a full life cycle assessment (LCA) of biomass-to-acetone as well as biomassto-ethanol, in the context of their co-production. LCA considered three scenarios: at the start of the project, best lab run, and best run data in combination with process integration and optimization. The largest contributions to the GHG emissions for the LanzaTech process are electricity consumption, whereas the largest negative contributors for the LanzaTech process is carbon sequestration in acetone when used in chemical applications. Using a combination of the best performance data in an integrated operation, the LanzaTech acetone process provided $\geq 180 \%$ GHG emission reduction (from cradle-to-gate) over the petrochemical (cumene) process. The LanzaTech ethanol process provided $90 \%$ GHG emission reduction (from cradle-to-grave) over

fossil fuel gasoline. This qualified LanzaTech ethanol as a cellulosic biofuel under the Renewable Fuel Standard ( $\geq 60 \%$ emissions reduction over fossil gasoline).

\section{Summary of Project Activities}

The goal of the project was addressed by integrating modeling, strain and pathway engineering and fermentation optimization, leading to commercially-viable production rate and selectivity (Figure 4). The technoeconomics and life cycle impacts were utilized to guide strain design and process optimization. 
Strain engineering

- Genome Scale Modelling

- Chassis strain construction

- Pathway engineering

Challenges:

- Limited enzyme variety

- Elimination of by-products

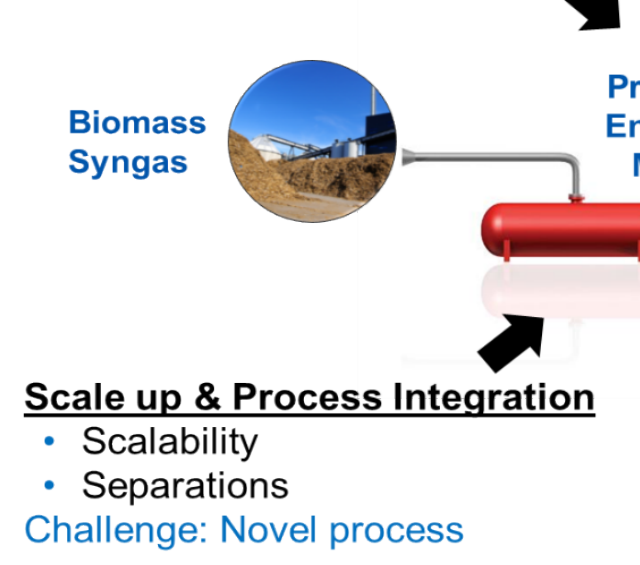

\section{Process development}

- Fermentation

- Omics

Challenge: Continuous process

Figure 4. Approach to project goals.

Genome scale model, strain engineering and fermentation optimization are discussed in Section 3.1, followed by the scale-up process in Section 3.2. LanzaTech strain collection whole genome sequencing and data mining are summarized in Section 3.3 followed by acetone pathway combinatorial analysis and synthetic pathway screening in Section 3.4. Omics analysis to answer key questions related to acetone production stability, strain behavior and correlation between transcripts and protein expression are summarized in Section 3.5. The TEA and LCA of the LanzaTech acetone process are summarized in Section 3.6. LanzaTech led the research conducted in Sections 3.1, 3.2, 3.3, 3.5 and 3.6. ORNL was the lead organization for systems biology studies (proteomics and metabolomics) described in Section 3.4.

\subsection{Genome Scale Model (GEM), Strain Engineering and Fermentation Optimization}

This section discusses research performed to build genome-scale models (GEMs) of acetone-producing strains to determine the best possible yields, growth rates and design landscape for each strain. Several iterative rounds of chassis strain constructions were carried out based on the GEMs predictions and a total of nine different chassis strain lines were constructed during the project. The performance of each strain was evaluated in bottles and the best performing strains were tested in a continuous fermentation in a continuous stirred-tank reactor (CSTR) and the results were fed back to GEMs to refine the models.

\subsubsection{GEM prediction of gene targets for improved acetone production}

To guide strain engineering we used constraint-based reconstruction and analysis (COBRA) methods to model $C$. autoethanogenum metabolism at a genome-scale. These techniques were used to identify gene knockout targets that would improve acetone yields, following the approach of O'Brien, Monk \& Palsson (2015) who provided an overview of genome- 
scale modelling in systems biology, including the mathematics, key assumptions and a summary of strengths and weaknesses ${ }^{11}$.

By modifying a $C$. autoethanogenum genome-scale model (GEM) LanzaTech has developed, similar to those described in Marcellin et al $^{12}$, and built GEMs of acetone-producing strains of $C$. autoethanogenum by adding heterologous genes and metabolic reactions to the model structure $^{8}$.

Using COBRA techniques including flux variability analysis, we interrogated the capabilities of these strains to determine possible yields, growth rates and the design landscape of each strain. We concluded that using a pathway with an enzyme that conserves the energy of thioester hydrolysis (CoA transferase or Ptb-Buk) would provide the best outcome. Flux balance analysis (FBA) demonstrated that while both options offer the same possible yields, the Ptb-Buk pathway would offer superior stability as acetone production could be decoupled from the production of the native fermentation products acetate and ethanol.

We carried out computational

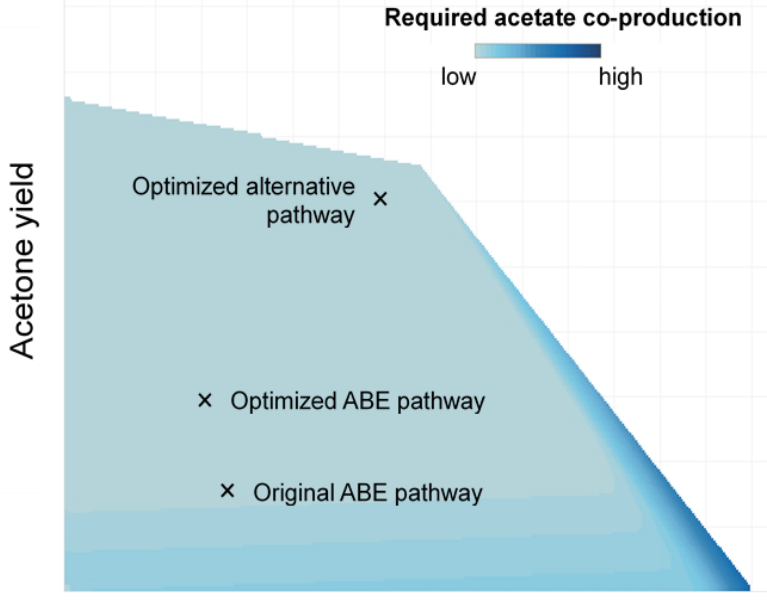

Growth rate

Figure 5. Production envelope showing the acetone yield/growth rate landscape for ATPgenerating acetone pathways, as well as predicted phenotypes for specific strains. strain optimization using evolutionary algorithms to identify a prioritized list of gene knockout targets predicted to improve acetone yields. We used FBA and minimization of metabolic adjustment to predict microbial phenotypes. Figure 5 illustrates some of these phenotypes in the context of the production envelope. We identified a growth-coupled strain design for the Ptb-Buk acetone production pathway, incorporating three gene disruptions. Provided the assumptions of the model were met, this strain was predicted to achieve $85.5 \%$ of the maximum possible acetone yield. We identified a nongrowth coupled strain design for the traditional $\mathrm{ABE}$ fermentation route, incorporating disruptions to three enzymes. This strain was predicted to achieve $38.7 \%$ of the maximum possible acetone yield. The model predicted that this yield could increase with fermentation optimization. Given that the metabolic network did not couple acetone production with growth, the model also predicted that the organism would operate in an unstable manner, with decreasing yields over time. Several other modifications to enhance acetone yields were also identified by the computational strain optimization algorithm.

When a chassis strain incorporating one of the predicted knockouts could not be successfully built, we carried out a second round of computational strain optimization. For this work, we constructed condition-specific genome-scale models using data generated from steady state gas fermentations. These data allowed us to determine a reference metabolic flux state that represented the behaviour of this strain during gas fermentation, allowing us to predict the impact of genetic perturbations more accurately. We selected a design with three additional disrupted 
enzymes. This strain was predicted to achieve $25.9 \%$ of the maximum acetone yield, but with scope for improvement through fermentation condition optimization.

This strain was successfully built and achieved significantly higher acetone yields than the baseline. However, bottle growth experiments indicated that one of the enzyme disruptions did not contribute to the improved acetone yields. We incorporated this information into the strain design algorithm, added recently-discovered enzyme promiscuity into the model and generated condition-specific models based on the latest fermentation data.

We subsequently carried out a third round of computational strain optimization and identified a set of disruptions to replace the previous target. Most of these targets were predicted to improve strain performance by reducing the production of an unwanted by-product 3hydroxybutyrate (3-HB), that was observed when the acetone pathway was added. A strain incorporating these knockouts was built and successfully achieved higher acetone yields; these high-performance production strains were further developed through fermentation condition optimization and the tuning of gene expression.

\subsubsection{Chassis strain construction, fermentation and feedback to GEMs}

C. autoethanogenum contains a secAdh gene encoding a primary:secondary alcohol dehydrogenase that converts acetone to isopropanol ${ }^{13}$. Therefore, the secAdh gene was our first gene knockout target for an acetone production strain. Before the project started in 2016, a secAdh interrupted strain was constructed using group II intron approaches (Clostron) ${ }^{14,15}$. The acetone pathway was expressed heterologously from a plasmid in the secAdh-interrupted strain, and acetone production was controlled by an inducible promoter, $\mathrm{P}_{t e t}{ }^{16}$. This strain was designated sAdh-0. After induction, sAdh- 0 showed combined acetone + ethanol productivity at $26.3 \%$ of the target commercial rate and acetone+ethanol co-selectivity at $90.6 \%$ of the commercial target, of which only $\sim 20.6 \%$ of the combined selectivity was acetone. In addition to the relatively low acetone production, the strain was also incapable of consuming hydrogen, a notable deficiency for a gas-fermenting production strain.

Therefore, one of the first items of the project was generating a new secAdh gene deletion mutant. The new strain was constructed using our proprietary homologous recombination-based method which can remove/insert gene(s) scarlessly ${ }^{17}$. In the presence of the acetone pathway, the new chassis strain (designated sAdh-1) was stable in a CSTR and was able to use hydrogen as an energy source. The sAdh- 1 strain demonstrated a combined acetone+ethanol productivity at $38 \%$ of commercial rate and co-selectivity at $91.1 \%$ of commercial target with stable acetone production for 4 days in a CSTR. Following GEM predictions and suggestions, two additional knockout strains (designated sAdh-2 and sAdh-3, respectively) were constructed consecutively to remove formation of the by-products 2,3-butanediol (2,3-BDO) and lactate. However, these strains were not stable in fermentation and were not pursued further.

Continued efforts updating and utilizing the GEM resulted in new predictions to promote acetone production. Three chassis strains (designated sAdh-4, sAdh-5 and sAdh-6, respectively) were constructed sequentially based on these modelling results with the aim of removing the byproduct 3-HB. The sAdh-5 and sAdh- 6 strains produced lower levels of $3-\mathrm{HB}$, at $10 \%$ and $5 \%$ of sAdh-1 levels, respectively. All three new chassis strains were stable in a CSTR and produced acetone and ethanol at similar rates and selectivities as their original parent strain. The acetone+ethanol co-productivity and co-selectivity achieved were $60 \%$ of the commercial rate and $94.7 \%$ of commercial combined selectivity, of which $49.3 \%$ of combined selectivity was from acetone. Both the rate and selectivity achieved and exceeded the Go/No-Go criteria of 
$>\mathbf{8 6 . 7 \%}$ of commercial selectivity and $50 \%$ of combined acetone and ethanol commercial rate, with $\mathbf{7 6 . 7 \%}$ of combined selectivity to acetone. With process optimization, we further demonstrated co-productivity of acetone+ethanol at $86.3 \%$ of commercial rate. The co-selectivity of acetone+ethanol was at $93.2 \%$ of commercial target with acetone selectivity at $81.5 \%$ of ethanol+acetone combined selectivity. The stability of acetone production improved from 3-4 days to 7 days.

Systems biology characterizations of the early acetone production strain (sAdh-0) (Section 3.5) identified that expression of the acetone pathway (expressed from a plasmid) decreased over time. In an attempt to address the decreasing expression, a new strain, designated Ace_1, with the acetone pathway integrated into the chromosome under a constitutive promoter was constructed based on the sAdh-4 parent strain. In CSTRs, the Ace_1 chassis strain had acetone+ethanol coproductivity at $78.3 \%$ of the commercial rate and co-selectivity at $96.8 \%$ of the commercial target with stability over 7 days. Further process optimization with the Ace_1 strain demonstrated that the strain can produce acetone+ethanol within $\sim 5 \%$ of the commercial rate and selectivity within $5 \%$ of the commercial target (this does not account for potential loss of the volatile acetone through the gas outlet in the laboratory set up). The Ace_1 strain had advantages over previous chassis strains in that it did not require antibiotics to maintain the acetone pathway and it did not require an antibiotic inducer for acetone production. Although we had met the DOE project milestone to construct a chassis strain with at least 3 KOs predicted by GEM in previous chassis strain constructions, we continued to improve the Ace_1 strain by deletion of genes encoding 3-HB pathway genes. 3-HB was found to be an unwanted by-product in this Ace_1 strain. We sequentially constructed Ace_2 and Ace_3 strains with further decreased 3-HB production in each strain. The acetone+ethanol co-production and co-selectivity for the Ace 3 strain were both very similar to that of the Ace_1 strain. A 20\% reduction of 3-HB level was observed for the Ace_2 compared to 3-HB produced in Ace_1, while over 95\% reduction was observed for the Ace_3 strain in a CSTR with 3-HB levels never above $0.1 \mathrm{~g} / \mathrm{L}$. Further process optimization of our current best strain Ace_3 is on-going, though outside the scope of the project.

\subsubsection{Summary}

We built GEMs to determine the best possible yields, growth rates and gene KO targets for acetone production strains. Based on GEMs as well as feedback from continuous fermentation experiments throughout the course of the project, we have constructed a total of nine different chassis strains. Of those nine strains, five were fully tested in CSTRs. Our end-of-project chassis strain (Ace_1) achieved within 5\% of our target acetone+ethanol co-productivity and coselectivity (not considering loss of volatile acetone through the gas outlet), without the major byproduct 2,3-BDO and was stable in CSTR for over 3 weeks, surpassing our goal of 7 days and also being antibiotic and inducer independent. A summary of chassis strains construction, progress, and by-product elimination is shown in Table 1. 


\begin{tabular}{|c|c|c|c|c|c|c|c|}
\hline $\begin{array}{l}\text { Acetone } \\
\text { pathway }\end{array}$ & $\begin{array}{l}\text { Gene } \\
\text { knock-outs }\end{array}$ & $\begin{array}{l}\text { Productivity } \\
\text { (g/L/h) }\end{array}$ & Byproducts & & Stability & Inducer & Antibiotic \\
\hline Plasmid & - & & 2,3-BDO & IPA & 3 days & Required & Required \\
\hline Plasmid & Adh KO & & 2,3-BDO & no $\mathrm{H}_{2}$ uptake & 3 days & Required & Required \\
\hline Plasmid & Adh KO* & & 2,3-BDO & & 1 week & Required & Required \\
\hline Plasmid & Adh $\mathrm{KO}^{*}+1$ & & 2,3-BDO & & 1 week & Required & Required \\
\hline Plasmid & Adh $\mathrm{KO}^{*}+2$ & & 2,3-BDO & & 1 week & Required & Required \\
\hline Integrated & Adh $\mathrm{KO}^{*}+2$ & & I & & $>3$ weeks & Not required & Not required \\
\hline Integrated & Adh $\mathrm{KO}^{*}+3$ & & & & $>3$ weeks & Not required & Not required \\
\hline
\end{tabular}

Table 1. Summary of constructed strains tested in CSTRs. The last five rows are strains constructed during this project.

Not only have we met our milestones in the construction of an acetone production chassis strain with at least three gene knockouts, we also demonstrated that genetic tools developed inhouse are sufficiently robust to manipulate the acetogen chromosome, which a decade ago was considered genetically intractable. Within the course of this project, we constructed strains with the largest number of modifications ever made in a single acetogenic Clostridium strain and generated acetone production strains ready for commercial deployment.

\subsection{Piloting Summary}

This section discusses the efforts made to scale the acetone+ethanol co-production process in an 80 -L scalable reactor, reasons that we did not reach the final milestones, and solutions to resolve issues discovered during the process.

\section{Pilot reactor runs}

Using our best performing acetone strain (Ace_1), over three 4-day + campaigns were conducted in the $80 \mathrm{~L}$ pilot reactor on syngas. Under comparable fermentation conditions and gas composition, the selectivity, productivity and stability in the $80 \mathrm{~L}$ pilot reactor were very similar to those obtained in the 2 L CSTR-based fermentation (Figure 6). Both the 80L pilot reactor (under 1.4 bar pressure) and 2 L CSTR-based fermentations produced similar titers of acetone, ethanol and biomass. The total selectivity and productivity were slightly better in the 2 L CSTR. This may have been due in part to the reactor configuration, particularly the condenser that was installed for the $2 \mathrm{~L} \mathrm{CSTR}$ to minimize loss of volatile acetone product. The similarity in performance between $2 \mathrm{~L}$ and $80 \mathrm{~L}$ reactors provides confidence that the acetone production strain and fermentation process are scalable, and the data can be transferred from lab scale to commercial scale. Furthermore, this was also the first time we demonstrated that the acetone production strain responded well when operated at elevated pressure, with the strain performing within the parameters previously observed in a 2 L nonpressurized reactor.

The $80 \mathrm{~L}$ pilot was not designed to work at the high gas flows that would be necessary to achieve the level of

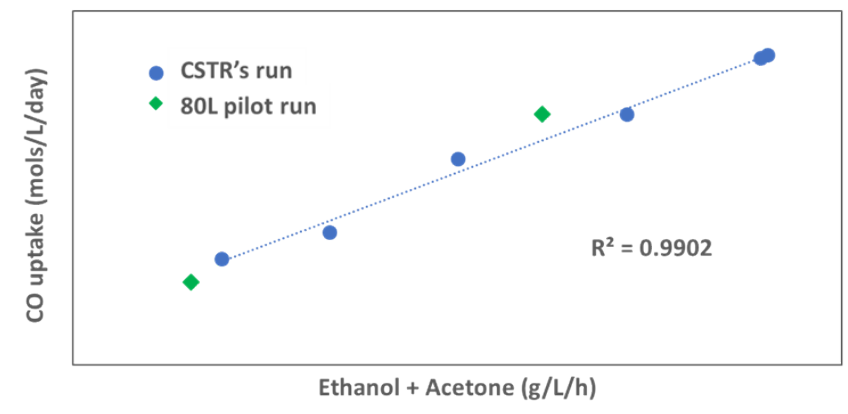

Figure 6. Good correlation between 2 L CSTR and $80 \mathrm{~L}$ Pilot runs 
productivity demonstrated in the 2 L CSTR set up. LanzaTech has previously demonstrated the scale-up of its commercial ethanol production process in a 20,000 L volume demonstration scale reactor and now to a $>500,000 \mathrm{~L}$ commercial reactor.

Based on the analysis and track record above, we have strong confidence that our acetone production process is both scalable and commercially viable. The next step is to re-configure the loop reactor at our pilot facility for high productivity operation, which is outside the scope of this project.

\subsection{Identification of Improved Enzymes by Genome Mining of Industrial Strain Collection}

JGI has in a separate effort (supported by DOE under Contract No. DE-AC02-05 CH11231) sequenced a total of 228 genomes of a collection of industrially deployed ABE Clostridia strains that LanzaTech co-owns and houses. The collection consists of over 300 Clostridia strains, mainly C. beijerinckii, $C$. saccharoperbutylicum and $C$. acetobutylicum, used in commercial ABE fermentation from 1944 to 1980 in South Africa and other commercial operations as well as various research and immunized strains. At the time of this report there are 228 genome sequences listed as complete, of which 209 genome sequences were generated using PacBio technology and the remaining 19 were generated using Illumina sequencing technology by the JGI.

In order to maximize the number of genomes sequenced and to access the potential diversity within the strain collection, a total of ninety-two gDNA samples that had failed PacBio QC were returned to ORNL and were sent to a third-party provider (Hudson Alpha) to generate Illumina Hiseq paired-end sequencing data for this project. Forty-five of these have not been PacBio sequenced and forty-seven ended up being duplicates of strains that later have been sequenced by PacBio and have been used to polish the PacBio sequences in house through hybrid assemblies. A total of 273 genome sequences for the industrial ABE clostridia strains have been generated, with 228 genomes sequences generated for individual strains by JGI and another 45 draft genome sequences generated using data from Hudson Alpha.

\section{Data mining (Data analysis and genotype-phenotype modelling)}

For this project, we were interested in the sequences for the acetone pathway genes (thlA, $c t f A, c t f B$ and $a d c$ ) as well as genes for LanzaTech's synthetic pathway, Ptb-Buk ( $p t b$ and buk). Sequences for these six genes were identified based on genome annotations and the sequences extracted from 200 completed genomes of the industrial ABE fermentation strain collection. Some of the strains coded for multiple copies of certain genes and some of the genes were missing from some strains. The total number of each gene of interest is listed in Table 2.

\begin{tabular}{|ccc|}
\hline Gene & $\begin{array}{c}\text { Total number found } \\
\text { in the collection }\end{array}$ & $\begin{array}{c}\text { Number of } \\
\text { unique sequences }\end{array}$ \\
\hline $\begin{array}{c}\text { Thiolase (ThlA) } \\
\text { CoA-transferase subunit A (CtfA) }\end{array}$ & 708 & 29 \\
\hline CoA-transferase subunit B (CtfB) & 213 & 9 \\
\hline $\begin{array}{c}\text { Acetoacetate decarboxylase (Adc) } \\
\text { Phosphate butyryl transferase } \\
\text { (Ptb) }\end{array}$ & 213 & 19 \\
\hline $\begin{array}{c}\text { Butyrate kinase (Buk) } \\
\text { Table 2. Summary of acetone pathway gene identified and mined from the } \\
\text { first 200 completed genomes of the industrial ABE fermentation strain } \\
\text { collection }\end{array}$ & 14 \\
\hline
\end{tabular}


In silico translated amino acid sequences of all identified genes were compared using alignment tools in the Geneious software package (Biomatters Ltd) and enzymes with unique amino acid sequences were identified (Table 2). The relationship of these unique amino acid sequences is represented in phylogenetic trees (Figure 7). The acetone pathway genes with unique amino acid sequences identified through this approach were applied in combinatorial analysis to screen for better acetone producing strains (Section 3.4.1) as well as to screen for Ptb-Buk variants that have improved selectivity towards acetoacetyl-CoA (Section 3.4.2).

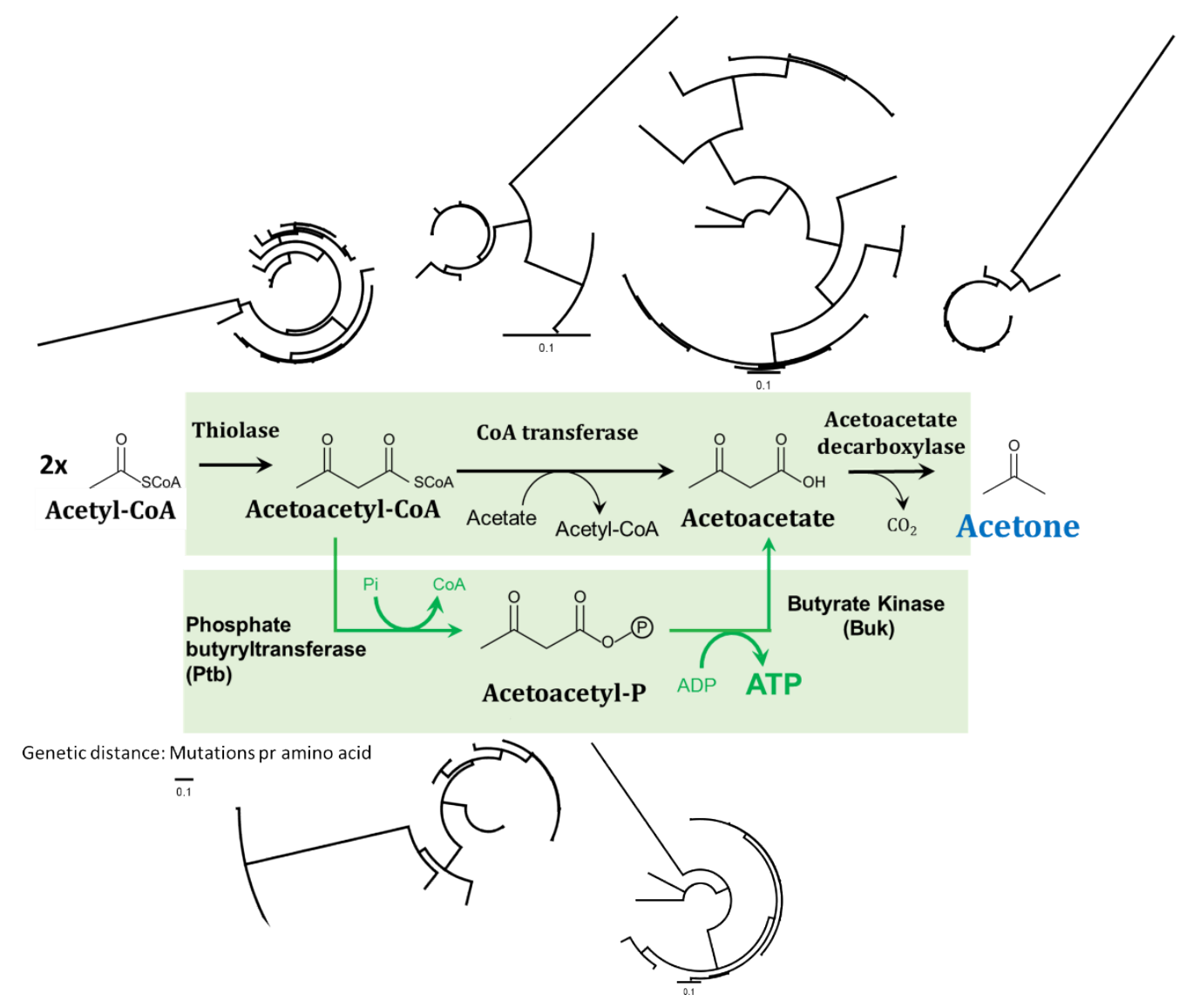

Figure 7. Phylogenetic trees for each gene of interest showing the relationship between the sequences

\subsection{Acetone Pathway Engineering and Optimization}

This section describes how the acetone pathway gene variants (thlA, ctfA, ctfB, adc coding for thiolase, CoA transferase subunit A, subunit B, and acetoacetate decarboxylase, respectively) identified from the industrial $\mathrm{ABE}$ fermentation strain collection in Section 3.3 were assembled and screened in order to identify the best combination for acetone production. We also discuss a platform to screen Ptb-Buk enzymes (phosphate butyryltransferase and butyrate kinase, respectively) with higher specificity towards acetoacetyl-CoA (immediate precursor of acetone) over acetyl-CoA (precursor of acetate). 


\subsubsection{Combinatorial analysis of acetone pathway variants and iterative pathway optimization}

In order to address bottlenecks and fine-tune expression for the acetone biosynthesis pathway, a combinatorial analysis was carried out. The analysis included four acetone pathway genes (thlA, ctfA-ctfB, and $a d c)$ and three Clostridial promoters of varying strength ( $\mathrm{P}_{\text {fer-laco-Us, }}$ $\mathrm{P}_{\mathrm{WL}}$ and $\left.\mathrm{P}_{\text {pfor }}\right)$. The three promoters, selected based on transcriptomic data and reporter gene assays, ranging from strongest $\left(\mathrm{P}_{\text {fer-laco-Us }}\right)$ to strong $\left(\mathrm{P}_{\mathrm{WL}}\right)$ to weak $\left(\mathrm{P}_{\mathrm{pfor}}\right)$. A hierarchical assembly approach was employed to first subclone each gene, terminator, (and promoter) into donor vectors using golden gate assembly, followed by a final golden gate assembly using recipient plasmids and the three donor vectors to obtain the acetone combinatorial library.

\begin{tabular}{|c|c|c|}
\hline thl $\boldsymbol{A}$ & $\boldsymbol{c t f} \boldsymbol{A}$-ctfB & $\boldsymbol{A d c}$ \\
\hline ThlA_1 & CtfAB_1 & Adc_1 \\
\hline ThlA_2 & CtfAB_2 & Adc 2 \\
\hline ThlA_3 & CtfAB_3 & Adc_3 \\
\hline ThlA_4 & CtfAB_4 & Adc_4 \\
\hline ThlA_5 & CtfAB_5 & Adc 5 \\
\hline \multirow{5}{*}{} & CtfAB_6 & Adc_6 \\
\cline { 2 - 3 } & CtfAB_7 & Adc_7 \\
\cline { 2 - 3 } & CtfAB_8 & Adc_8 \\
\cline { 2 - 3 } & CtfAB_9 & Adc_9 \\
\cline { 2 - 3 } & CtfAB_10 & Adc_10 \\
\cline { 2 - 3 } & CtfAB_11 & Adc_11 \\
\cline { 2 - 3 } & CtfAB_12 & Adc_12 \\
\cline { 2 - 3 } & CtfAB_13 & \multicolumn{1}{|c}{} \\
\cline { 2 - 3 } & &
\end{tabular}

Table 3. Acetone pathway genes included in the targeted acetone combinatorial library

With the aim of identifying enzyme variant combinations with superior acetone production, thlA, $c t f A-c t f B$, and $a d c$ genes from the industrial $\mathrm{ABE}$ fermentation strain collection were included in the combinatorial analysis. In the first instance, degenerate primers were used to amplify acetone pathway genes from genomic DNA of the industrial ABE fermentation strain collection before assembly into the donor vectors. Using only one set of degenerate primers per acetone pathway gene, $65 \%$ of $t h l A, 69 \%$ of $c t f A-c t f B$ and $93 \%$ of $a d c$ were successfully amplified from the collection. Both pooled and un-pooled acetone combinatorial libraries were transformed into the sAdh-4 strain (Section 3.1.2). Sequencing analysis of the resultant strains showed a high frequency of frameshift mutations in the $5^{\prime}$ and $3^{\prime}$ end of the acetone pathway genes, most likely due to the degenerate nature of the primers.

To obtain a combinatorial library with intact acetone pathway genes, a more targeted approach was undertaken. A total of 5 thlA, 13 ctfA-ctfB and 12 adc genes (Table 3), each of different amino acid sequence, were amplified using specific primers before assembly into donor vectors. When combined with the three non-repetitive promoters, the theoretical permutation was $3 \times 5 \times 2 \times 13 \times 1 \times 12=4680$. Following assembly in Escherichia coli, thousands of colonies were obtained; PCR screening and restriction digest analysis were carried out to check the integrity of the acetone combinatorial library. Sequencing of the plasmids showed good levels of gene variant diversity and significantly lower frequency of mutations in the 5 ' and $3{ }^{\circ}$ of acetone pathway genes, confirming the hypothesis that the use of degenerate primers resulted in high levels of mutations at these regions.

The combinatorial library was introduced into sAdh-4 via conjugation. In total, 236 combinatorial strains (which is $>10 \mathrm{x}$ the size of any published acetogenic dataset) were screened for acetone production in Schott bottles with defined minimal medium using a high-CO gas blend. Following 8 days of incubation, biomass concentration was measured spectroscopically, and metabolites were analyzed using HPLC. A wide range of acetone titer was observed with up to 10x increased production over the initial construct employing genes from the public repository (Figure 8).

More detailed growth curves for 50 of the 236 combinatorial strains were obtained in Schott bottles (same conditions as above) to verify the acetone production in these strains. 
Twelve of these strains (ranging from low to high acetone producers) were also tested in CSTR. In order to determine whether the bottle screening results were consistent with performance (in terms of acetone production) in CSTRs, a correlation of acetone titers between titers observed in bottles and CSTRs was established. The method was

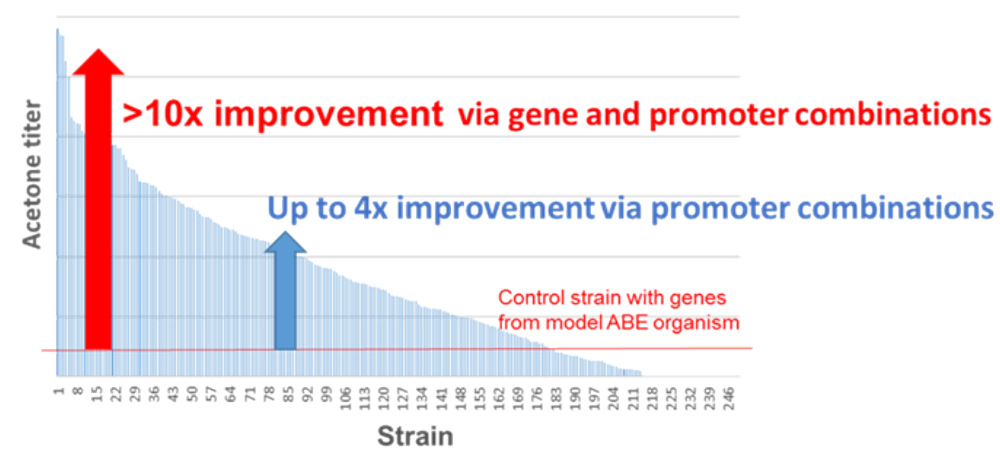

Figure 8. Distribution of end-point acetone titer by acetone combinatorial strains.

able to successfully identify highest acetone producers (with high correlation between batch growth in bottles and continuous fermentation in CSTRs) as well as low acetone producers, though correlation in between varied. This dataset suggested that a growth experiment at the bottle scale is a valid screening method for acetone producing strains.

In conclusion, a hierarchical assembly method was developed to generate an acetone combinatorial library with different promoters and acetone pathway genes from the industrial ABE fermentation strain collection. A total of 236 acetone combinatorial strains were characterized at the bottle scale and 12 were subjected to CSTR runs. A wide range of acetone production titer was observed in the combinatorial library. Compared to the initial design with genes from the $C$. acetobutylicum type strain ATCC824, a >10x improvement in acetone titer was observed.

\subsubsection{Platform established to screen and select Ptb-Buk variants in vitro and in vivo}

\section{Development of an enzyme assay for screening Ptb-Buk variants in vitro}

Preliminary LanzaTech experiments demonstrated that the Ptb-Buk enzyme pair can be functionally expressed in C. autoethanogenum. Ptb-Buk catalyses the conversion of acetoacetylCoA to acetoacetate, the immediate precursor of acetone, and generates one net ATP per reaction. However, substrate specificity of Ptb-Buk towards acetoacetyl-CoA has been documented as being poor (the preferred substrate is butyryl-CoA). An additional complication of Ptb-Buk is that it is

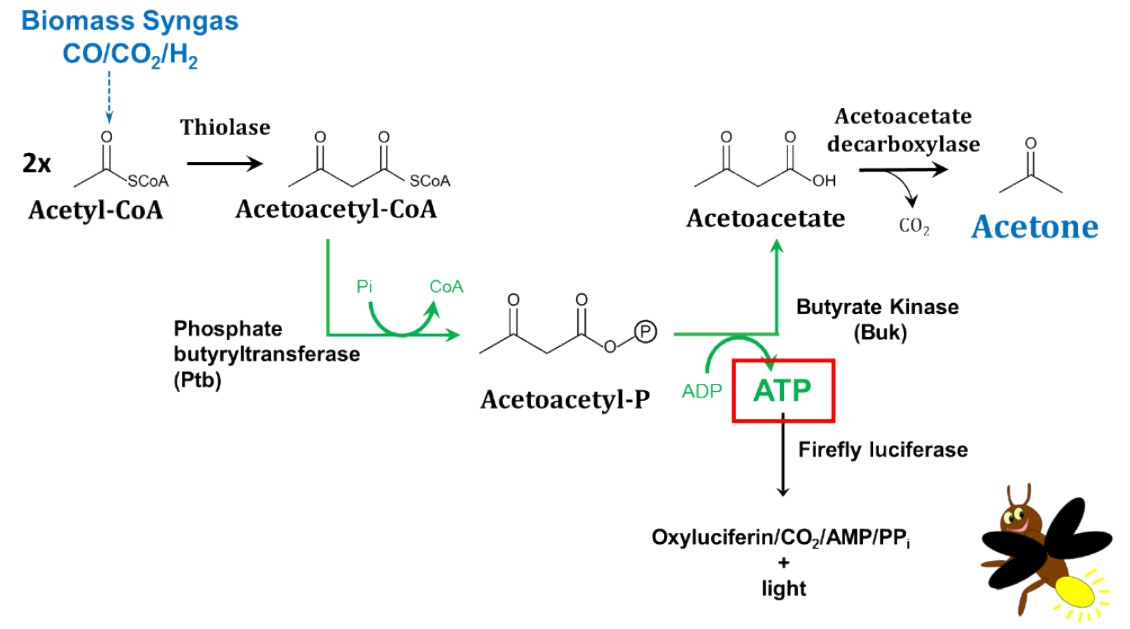

Figure 9. Using luminescence to screen for ATP production via Ptb-Buk reaction 
also capable of catalysing the conversion of acetyl-CoA, the precursor to acetoacetyl-CoA, to acetate, which would reduce flux to acetone. We developed a screening protocol to identify a PtbBuk system with improved substrate specificity towards acetoacetyl-CoA over acetyl-CoA. The ptb-buk gene sequences were identified from either the public databases or from the industrial ABE fermentation strain collection.

The assay screens for ATP production by Ptb-Buk were coupled with luminescence by firefly luciferase (Figure 9). Generally, the higher the intensity of bioluminescence produced in the presence of a given substrate, the more ATP generated, and, therefore, greater conversion of that CoAbearing substrate to its corresponding carboxylic acid. A total of 20 Ptb-Buk pairs were assayed, and the data are summarized in Figure 10. Of the Ptb-Buk pairs tested, 5 (green bars in Figure 10) had average specificities towards acetoacetylCoA over acetyl-CoA of at least $50 \%$ greater than the comparison pair (Ptb-Buk from $C$. beijerinckii).

Based on our enzyme assays results, selected Ptb-Buk pairs have been moved into a $C$. autoethanogenum expression system described in Section 3.4.1 (ptb-buk genes replacing $c t f A B$ ).

\subsection{Systems Approach to Identify Bottleneck in Current Fermentations}

This section discusses using omics analyses to understand challenges encountered during the project, including acetone production instability, strain stability, and acetone stress responses etc. Correlations between acetone production, protein and RNA expression levels are also discussed in this section. ORNL developed a mass spectrometry-based method to quantify energy metabolites and, especially, Coenzyme A (CoA) substrates to further the understanding of metabolic responses in terms of acetone production.

\subsubsection{Challenge: Overcome acetone production instability}

The acetone-producing strain (sAdh-0, Section 3.1.2) that was constructed before the start of the project was unstable in producing acetone: the acetone titer peaked three days after induction and slowly decreased over time, thereafter. To identify the reasons behind the unstable acetone production, three technical replicates of fermentation runs were carried out and a total of 35 samples from 12 time points were collected over the fermentation runs for transcriptomics, proteomics, and metabolomics measurements.

An average of over five million reads per sample were produced from the RNAseq runs. An average of $97 \%$ reads were mapped to the reference genome sequence, indicating good quality data. Two different analysis packages, cuffdiff $2^{18}$ and DESeq $2^{19}$, were used to analyse the data. 
A total of 1997 genes were differentially expressed at some point over the fermentation runs. Based on gene expression patterns throughout the fermentations, a total of eight clusters of gene expression patterns were identified. Most notably, the expression of the acetone pathway genes (thl, $c t f A, c t f B$ and $a d c)$ are within one pattern that declined concurrently with acetone level in the fermentation runs (Figure 11).

In contrast, the transcript of the tet $R$ gene, which codes for a repressor that inhibits the expression of acetone pathway genes in the absence of an inducer (sAdh-0, Section 3.1.2), along with the transcripts of other housekeeping genes on the plasmid were found to be at a stable level throughout the fermentation runs (data not shown). All major metabolite pathway genes, such as acetate, ethanol, 2,3-BDO and lactate were stable throughout the fermentation runs. The Wood-Ljungdahl pathway and ATP synthase gene expression were also stable over the course of the experiment. Expressions of the Rnf complex and Nfn complex genes, involved in energy metabolism and redox balance ${ }^{20,21}$, were decreased over time (data not shown).

Proteomics measurements showed consistency across all LC-MS runs. In total, 1779 proteins were identified across 1.22 million mass spectra for all samples from 21,592 distinct peptide sequences. ANOVA identified 545 proteins that differed in abundance at some point over the fermentation runs. Protein abundance trends were clustered along with the acetone trend over the entire time series and during acetone's fall from peak concentration. Proteomics data were in agreement with the transcriptomic data, with

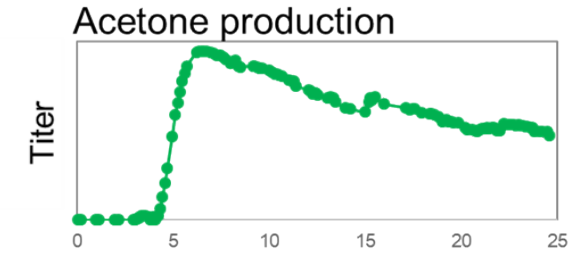

Transcriptome
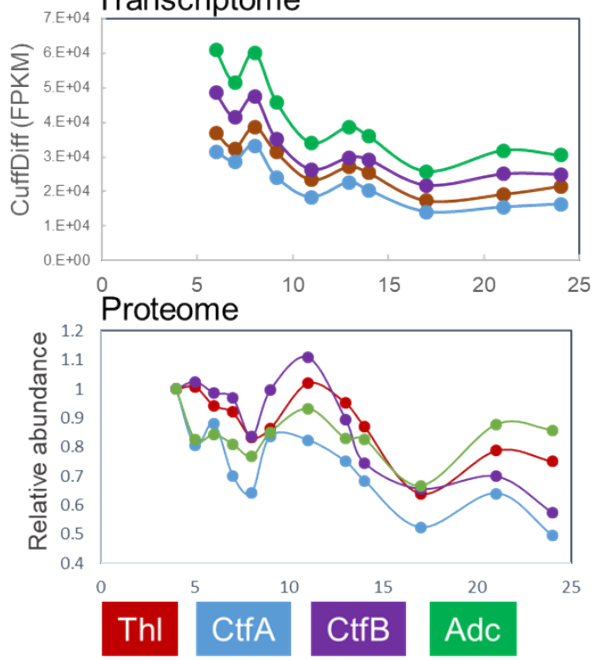

Figure 11. Acetone production decreased over time in correlation with gene/protein expression data. acetone pathway protein levels decreasing over time, while the controls were stable over the fermentation runs (Figure 11).

In addition, proteins with decreased abundance over time included various $\mathrm{ABC}$ transporters related to molybdenum/molybdopterin metabolism, a chaperone protein Hsp20, an exopolysaccharide biosynthesis protein and proteins involved in chemotaxis and/or environmental sensing, and glyoxylase, an enzyme that carries out the detoxification of methylglyoxal and the other reactive aldehydes to less cytotoxic intermediates. Up-regulated proteins included those involved in cys/met transport and metabolism/sulfur metabolism (cysteine synthase and cystathionine gamma-synthase, NLPA lipoprotein, serine acetyl-transferase, disulphide oxidoreductase, glutamate synthase), a propanediol utilization protein, and an aldehyde:ferredoxin oxidoreductase (AOR) (converts acetate to ethanol $)^{20}$.

With regard to metabolomic measurements, extracted metabolites were derivatized by trimethylsilylation and analysed by gas chromatography-mass spectrometry (GCMS) using electron impact ionization $(70 \mathrm{eV})$. Over 100 of the most abundant peaks were quantified, corresponding to a total of 97 intracellular metabolites, with the most abundant metabolites being alanine, 3-hydroxybutyrate, glycerol-1/3-phosphate, an unidentified metabolite at retention time 
9.77 min with key mass-to-charge (m/z) 129203117 291, aspartic acid, and glutamic acid. The metabolite concentrations were correlated with acetone production. Those metabolites negatively correlated with acetone production included several partially identified indoles, meso-2,3butanediol $^{22}$, 2-hydroxy-2-methylbutyric acid, 2-hydroxybutanoic acid, and 4-hydroxybutanoic acid. For due diligence, we confirmed that our target microbial species does not synthesize sorbitol, the key metabolite that we use as an internal standard for all of our GCMS-based metabolomics measurements.

Based on these results, we integrated the relevant pathway genes into the chromosome to improve acetone production stability. This has resulted in our best chassis strains (Ace_1, Ace_2 and Ace_3 in Section 3.1, Table 1).

\subsubsection{Understanding cellular response to acetone stress}

An experiment was conducted to understand what cellular responses were triggered by acetone challenge on autotrophic growth using wild type $C$. autoethanogenum as the host microorganism. An acetone minimal inhibitory growth experiment was first carried out in order to determine the toxicity level of acetone in bottles. Even though the closed bottle set up is not representative of the open, continuous CSTR set up where acetone is stripped, we choose this system to be able to screen more concentration differences. Slowed bottle cell growth was observed with acetone concentration at $15 \mathrm{~g} / \mathrm{L}$ and $30 \mathrm{~g} / \mathrm{L}$ (Figure 12). Higher acetone concentrations up to $80 \mathrm{~g} / \mathrm{L}$ were also tested, but these concentrations had completely inhibited cell growth (data not shown). Therefore, acetone concentrations higher than $30 \mathrm{~g} / \mathrm{L}$ were not considered for further testing. Triplicate growth in bottles was carried out with different concentrations of acetone $(15 \mathrm{~g} / \mathrm{L}$ and $30 \mathrm{~g} / \mathrm{L}$ ) added at the early log growth phase, and then cells were incubated for another 48 hours. Non-stressed cultures that served as controls were incubated along with acetone stressed cultures. Samples were collected for RNAseq, proteomics, and metabolomics analysis at time $0 \mathrm{~h}$ and again at $48 \mathrm{~h}$ incubation of acetone stress. Due to low cell biomass and low sampling volume, we were not able to obtain good quality metabolomic data, but we were able to obtain good quality RNAseq and proteomics data.

Under acetone stress conditions, higher level expression of GroEL (molecular chaperones), heatshock proteins, including GrpE, alkaline shock protein and others, were detected. This was expected as these proteins are known to improve protein folding integrity when cells are under duress. Another group of genes/proteins with higher expression were transcriptional regulators. This may indicate that cells were going through physical responses to acetone stress and up/down-regulating gene expression in response to acetone stress. Other proteins with higher expression levels included butanediol dehydrogenase, butanol dehydrogenase, molybdopterin-binding protein, xanthine dehydrogenase, and an aldo/keto reductase.

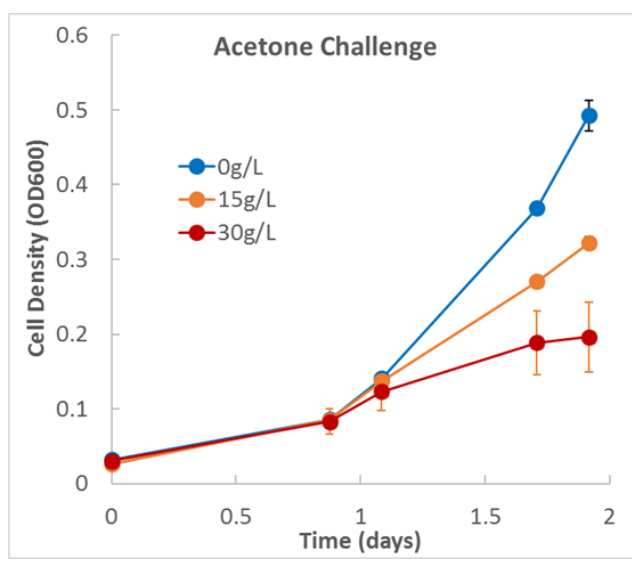

Figure 12. Minimal inhibitory concentration of acetone on cell growth 


\subsubsection{Development of mass-spectrometry-based approach to characterize energy metabolites}

In order to better understand metabolic activities and regulation for microbially-based acetone production, we sought to examine and implement a mass spectrometry-based approach for characterizing redox metabolites, such as NAD, NADH, NADP, NADPH, ATP, ADP, and the different Coenzyme A (CoA) intermediates (acetyl, acetoacetyl and hydroxybutyryl-CoA). Our goal was to utilize a liquid chromatography tandem mass spectrometry (LC-MS/MS) approach to both detect and quantify these metabolites in a fashion similar to the existing proteome measurements in fermentation samples. Initial work was directed at examining pure standards of these redox metabolites in an effort to examine MS response/sensitivity/mass accuracy/fragmentation pathways. We were able to get high mass accuracy measurements (less than 1 part-per-million) on the parent ions in negative ion mode for all of these compounds. Extensive fragmentation information was acquired for each compound as well. Initial work using pure standards of these redox metabolites highlighted their lability due to multiple freeze/thaw cycles and sample aging. We thus developed a quick and simple protocol using a 50\%-50\% water-methanol mixture to efficiently extract the metabolites directly from frozen microbial cells, both minimizing time-dependent degradation and avoiding freeze/thaw cycles altogether. LC-MS measurements were made immediately after fresh extraction using 1D reversedphase liquid chromatography coupled to a QExactive+ mass spectrometer. We were able to detect all energy metabolites simultaneously in
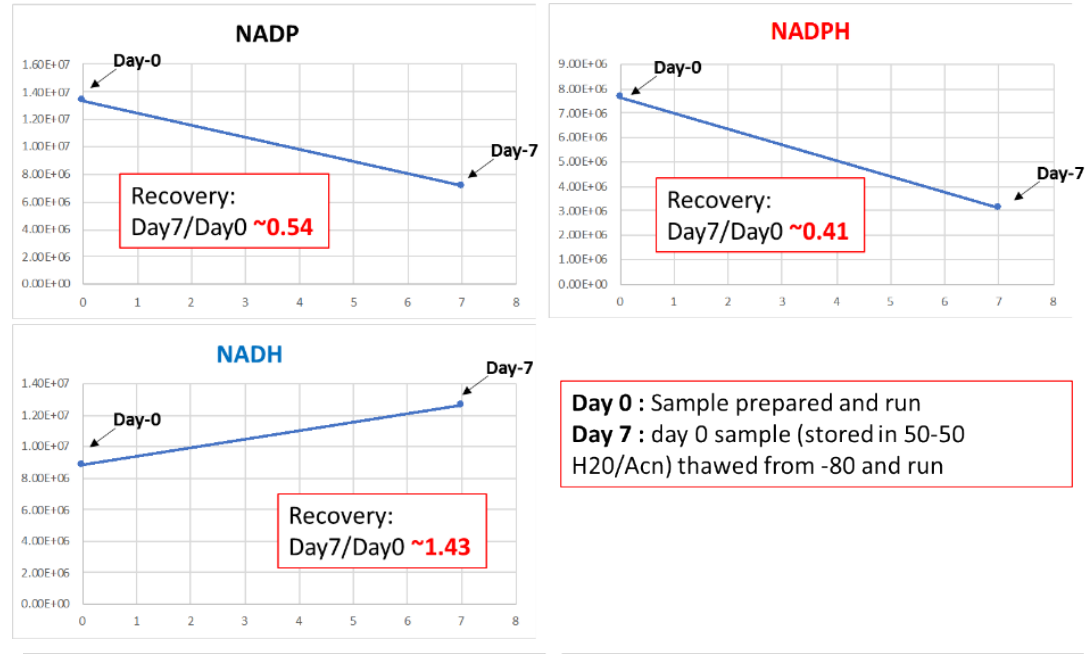

Day 0 : Sample prepared and run Day 7 : day 0 sample (stored in $50-50$ $\mathrm{H} 20 / \mathrm{Acn}$ ) thawed from -80 and run

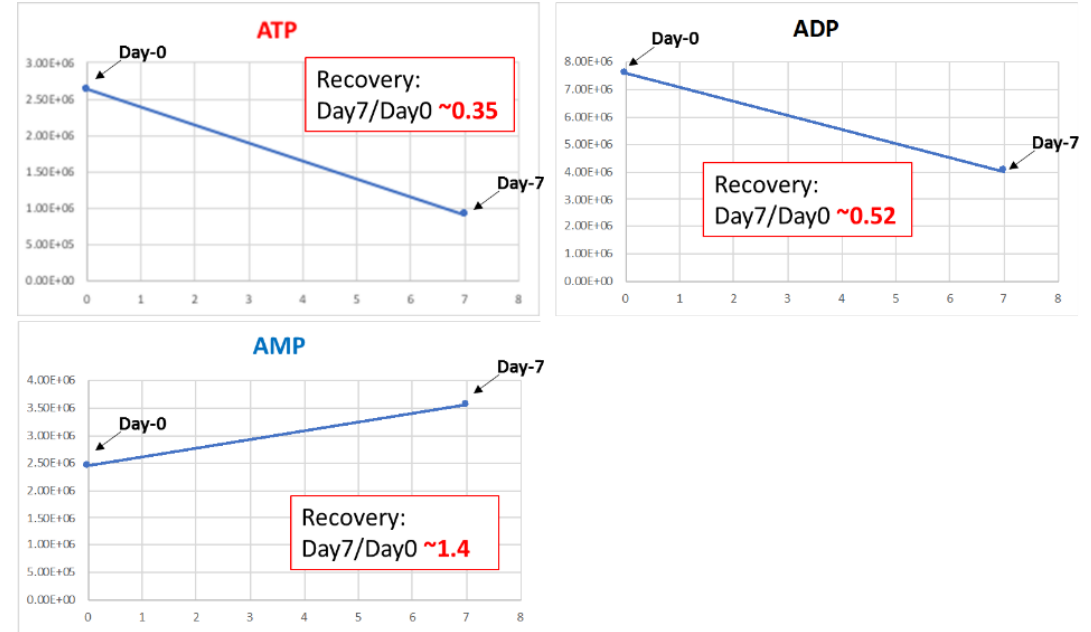

Figure 13. Stability of NADPH, NADP, NADH and AXP a single run for each sample. Extracted ion chromatograms for each metabolite were derived and abundance trends plotted across samples.

In order to better understand stabilities of these fairly labile metabolites, we prepared stock solutions of each compound, and then stored them at $-80{ }^{\circ} \mathrm{C}$ for periods of time ranging from 1 day to 7 days. We found that AMP and NADH were relatively stable over this time-frame, NAD, NADP, and ADP were moderate in stability, but ATP and NADPH were quite unstable, both degrading to about 50\% signal response over this time period (Figure 13). 
The methods developed here were applied to fermentation samples to better understand the relationships between acetone production and energy metabolites.

\subsection{Technoeconomic and Lifecycle Analysis}

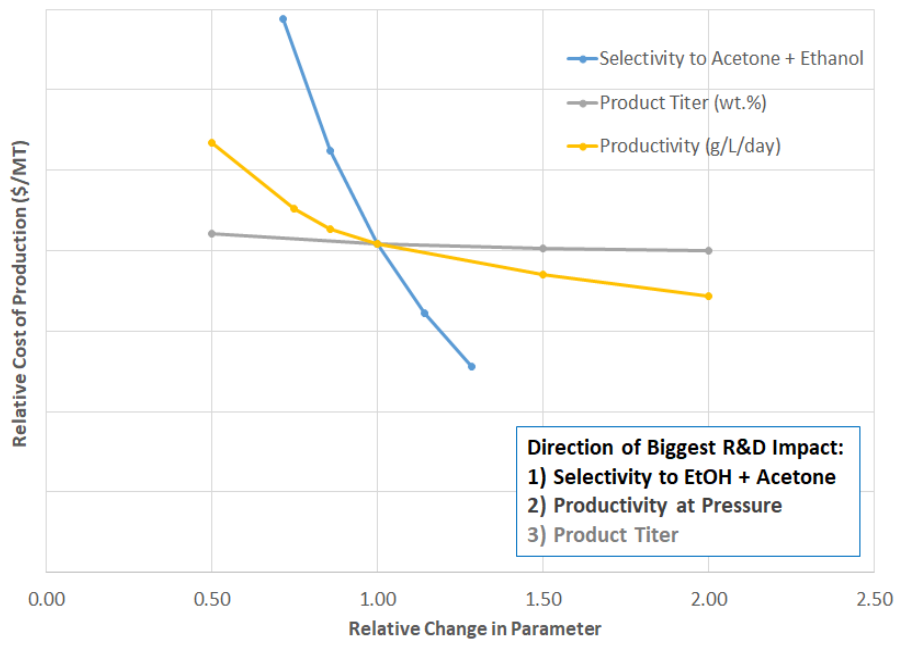

Figure 14. Technoeconomic Tradeoffs-Variation of Single Parameters. 
This section discusses (1) technoeconomic analyses carried out throughout the project to guide the overall strategic direction of the project, assess the economic and life cycle impacts, and inform the post project commercialization strategy; and (2) Greenhouse gas (GHG) emissions comparisons between the LanzaTech acetone process and the traditional petrochemical (cumene) process. To summarize and as detailed below, acetone selectivity and productivity have greater impact on the cost of production compared to product titer (Figure 14). With the agreement of DOE, the goals and milestones were changed accordingly to pursue co-production of acetone + ethanol with high selectivity and productivity instead of titer. The cash cost of production (CCOP) was also calculated for each strain developed over the course of the project and compared to current market value of acetone in the United States in order to show progress toward a commercially viable process.

\subsubsection{Summary of technoeconomic analysis}

In order to focus research and efforts in the highest impact areas, a technoeconomic sensitivity analysis was constructed for the acetone fermentation process examining three core fermentation parameters: selectivity to acetone as a percentage of products, selectivity to acetone + ethanol, productivity, and acetone and ethanol titer (Figure 14). The technoeconomic analysis (TEA) was based on the conversion of biomass derived syngas into ethanol and acetone using results from laboratory data obtained throughout the project. Specifically, we analyzed fermentation data during/from key developments throughout the project, from the start to the pilot run. The process configuration consists of indirect gasification based on the NREL process (NREL/TP-5100-47594) of 2000 bone dry metric tonnes (MT) per day of biomass. Capital and utility costs in the TEA were assessed on a 2015 US Gulf coast basis. At the start of the project (Figure 15, 1), the cash cost of production (CCOP) was $>2.7 \mathrm{X}$ higher than our commercial target (Figure 15, 6). With each set of strain improvement, a decrease of CCOP was achieved (Figure 15, 3, 4, 5). At the end of the project, with fermentation data from our best chassis strain (Figure $15,5)$, the CCOP was only $6 \%$ higher compared to our target.

A TEA analysis was also carried out based on the $80-\mathrm{L}$ pilot reactor runs and compared against the results from previous runs including the Go/No-Go milestone (Figure 15, 2) and the final target runs (Figure 15, 6). We also did a TEA analysis based on fermentation trials with further process optimization which indicated that low acetate selectivity is an outcome of this

operational mode. This modelling allowed us to understand the economic impact for similar pilot run conditions since we do not have the ability to demonstrate the effect of the particular process

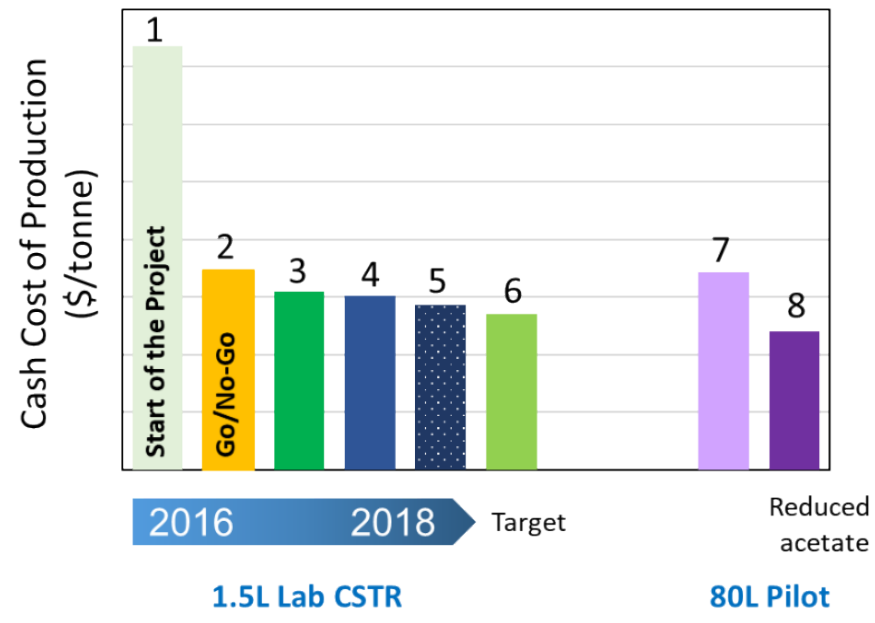

Figure 15. Decrease in Cash Cost of Production over the course of the project.

optimization in the current system. The production economics are expected to improve with process optimization and a closed loop system (Figure 15,8). LanzaTech has demonstrated the 
same process optimization at a commercial ethanol production facility, and it is expected that this result for an acetone-ethanol product system can be achieved with the appropriate equipment and process configuration.

\subsubsection{Summary of Life Cycle Analysis}

The commercial-scale GHG emissions were calculated for ethanol and acetone produced from gasified forest biomass residues using gas fermentation. The commercial production process occurs in multiple steps, starting with the collection of forest residues, followed by biomass transportation to the biorefinery, gasification, and finally gas-fermentation to the two co-products (ethanol and acetone). The system boundary for the life-cycle analysis includes all material and energy inputs for these steps. The GHG emissions for biomass collection/transport and gasification were taken from the most recent version of the GREET 1 Model (available at: https:/greet.es.anl.gov/), as well as the emission factors for electricity, steam, and chemicals used in the conversion process. GHG emissions were allocated to the two co-products based on their energy content. The boundary of analysis for acetone stops at production, so a credit is taken for the carbon sequestered in the chemical for both syngas- and petroleum-derived cases. However, as we expect ethanol to be used as a fuel, its boundary extends to fuel combustion.

The GHG emissions were calculated for commercial designs based on the following sets of experimental data: at the Start of the project, and at the End of the project. The data collected at the Start of the project served as the baseline of the analysis. The other set was based on our best result derived from running the integrated strain (Ace_1). In addition to these scenarios, we also considered ways to reduce the emissions further by evaluating two scenarios using the dataset derived at the end of the project: (Case A) heat integration to meet process utility requirements, and (Case B) chemical recycling or using alternate chemicals with different GHG profiles. In summary, the GHG emissions were evaluated for all scenarios and compared to the fossil baseline case. The scenarios evaluated were:

- Start: Process parameters at the start of the project (productivity: $26.3 \%$ commercial rate, combined selectivity of ethanol plus acetone: $90.6 \%$ of commercial target, of which $20.6 \%$ was from acetone)

- End: Commercial design based on the best experimental run at the end of the project (Ace_1) (combined acetone+ethanol productivity: 95.3\% commercial rate, combined selectivity of ethanol plus acetone: $94.7 \%$ commercial target, of which $57.3 \%$ was from acetone)

- Case A - Heat integration: End scenario redesigned to use heat integration.

- Case B - Chemical recycling or Alt chemicals: End scenario redesigned to use alternate chemicals/chemical recycling.

- Gasoline/Conventional: Fossil gasoline and acetone used as a comparator. 
The calculated GHG emissions for all scenarios are provided in Figure 16A and 16B for acetone and ethanol, respectively. At the Start of the project, the acetone emissions were -0.15 $\mathrm{kg}-\mathrm{CO}_{2} \mathrm{e} / \mathrm{kg}$ acetone and decreased to $-1.35 \mathrm{~kg}-\mathrm{CO}_{2} \mathrm{e} / \mathrm{kg}$ acetone at the end of the project (Figure 16A). The largest contributions to the $\mathrm{GHG}$ emissions are electricity consumption, which was modeled using the U.S. average grid mix, steam use, and chemicals use. The credit for the carbon sequestered in the acetone is also a large (negative) contributor to the emissions. Heat integration in Case A provides the lowest GHG emissions at $2.07 \mathrm{~kg}-\mathrm{CO}_{2} \mathrm{e} / \mathrm{kg}$ acetone. However, Case $\mathrm{B}$ has emissions of $-1.28 \quad \mathrm{~kg}-\mathrm{CO}_{2} \mathrm{e} / \mathrm{kg}$ acetone by comparison. These results were compared to the GHG emissions of conventional acetone (from the GREET 1 model) to evaluate the potential emissions savings. The boundary for the conventional chemical also only goes through acetone production, to make a consistent comparison. At the Start of the project, producing acetone from conversion of gasified forest residues using LanzaTech's gas fermentation technology would result in a GHG savings of $2.70 \mathrm{~kg}-\mathrm{CO}_{2} \mathrm{e} / \mathrm{kg}$ acetone, whereas at the end project all scenarios would result in GHG savings of 3.83 to $4.62 \mathrm{~kg}$ $\mathrm{CO}_{2} \mathrm{e} / \mathrm{kg}$ acetone.

As with acetone, electricity consumption,
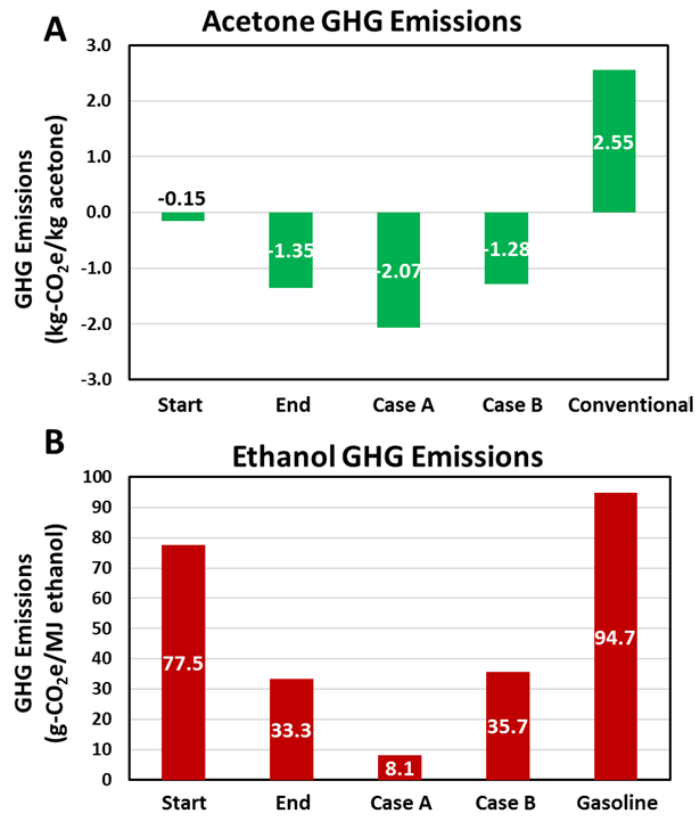

Figure 16. LanzaTech process provides GHG emissions savings over traditional petrochemical process: (A) Acetone; (B) Ethanol GHG emissions. steam use and chemical use are the largest contributors to the emissions of ethanol co-production. The GHG emissions for ethanol at the Start of the project were $77.5 \mathrm{~g}-\mathrm{CO}_{2} \mathrm{e} / \mathrm{MJ}$ (Figure 16B). At the End of the project, the emissions were reduced to $33.3 \mathrm{~g}-\mathrm{CO}_{2} \mathrm{e} / \mathrm{MJ}$. Case $\mathrm{A}$ provides the lowest GHG emissions at $8.1 \mathrm{~g}-\mathrm{CO}_{2} \mathrm{e} / \mathrm{MJ}$, while Case B increases the GHG emissions to $35.7 \mathrm{~g}-$ $\mathrm{CO}_{2} \mathrm{e} / \mathrm{MJ}$. These results were compared to the GHG emissions of fossil gasoline (from the GREET model) at 94.7 g- $\mathrm{CO}_{2} \mathrm{e} / \mathrm{MJ}$ gasoline. Case A scenario provides the largest reduction at $91 \%$, while the reduction for Case B is $62 \%$. At the Start of the project, the ethanol only provided an $18 \%$ emissions savings and would not qualify as a cellulosic biofuel (minimum $60 \%$ GHG reduction) or under the Renewable Fuel Standard (https://www.epa.gov/renewable-fuel-standardprogram/overview-renewable-fuel-standard).

Overall, our current best run results combined with process integration and optimization, shows that the LanzaTech acetone process provides a GHG emissions savings of $4.62 \mathrm{~kg}-\mathrm{CO}_{2} \mathrm{e} / \mathrm{kg}$ acetone over fossil-derived acetone ( $\geq 180 \%$ GHG emissions savings over conventional process). The same best run scenario also produces an ethanol co-product with a $91 \%$ GHG emission savings over fossil gasoline. This would qualify LanzaTech ethanol as a cellulosic biofuel under the Renewable Fuel Standard ( $\geq 60 \%$ emissions reduction over fossil gasoline).

\subsection{Technology Transfer and Commercialization Activities}

\section{Subject Inventions (As defined in the CRADA)}


None.

\section{Commercialization Possibilities}

\section{Patent Applications and Licensing Agreements}

LanzaTech has background IP related to the production of acetone and isopropanol, as described in International Patent Application Publication No. WO 2012/115527.

\section{Commercialization}

Acetone Market

Acetone has a number of important downstream products including drop-in fuels, fuel additives and important commodity chemicals that represent significant demand beyond the current uses for acetone:

- Jet-fuel (via direct extractive fermentation) - a drop-in jet fuel for aviation industry.

- Isobutylene (direct fermentative production) - used for synthesis of fuel additives and end products such as synthetic rubber, with an annual global market size of US\$25-29 billion. Beyond its use in chemistry and as a fuel additive (15Mt/yr)

- Isooctance (by catalytic upgrading of isobutylene) - a high performance, drop-in fuel for standard gasoline cars.

- Isopropanol (direct fermentative production) - important solvent with a market of US\$2.53.5 billions growing at $2.5 \%$ per year.

- Propylene (by catalytic upgrading of isopropanol) - the second-largest volume petrochemical, with a global market of over US\$125 billion that is growing at $5.0 \%$ per annum

- Bisphenol A (BPA) (by catalytic upgrading of acetone) - Important polymer (e.g. polymers or epoxy resins) with a market of US\$10 billion

- Polmethyl methacrylate (PMMA) (by catalytic upgrading of acetone) - Important polymer (e.g. plexiglas) with a fast growing US\$7 billion market

Acetone is a by-product of the cumene process, the main driver of which is phenol production. There are two major market forces which have the potential to impact acetone prices:

- The growth of Bisphenol A production increases demand for phenol relative to acetone, as it requires two phenol molecules for every acetone molecule. This has the potential to decrease the price of acetone.

- The development of alternative phenol production routes could decrease the supply of acetone. There are two main processes in development, the commercialization of which would increase the price of acetone:

$\circ$ The direct oxidation of benzene to create phenol; and

- Cleaving sec-butylbenzene (SBB) to produce phenol and methylethylketone (MEK).

Due to its status as a by-product, acetone prices fluctuate significantly. In China, the largest global market for acetone, prices reached a high of almost $\$ 1,600$ per tonne in 2013, collapsed to $\$ 540$ per tonne in 2015 , recovered to $\$ 1,050$ per tonne in 2016 , and fell to $\$ 567$ per tonne in 2018 . The development of a non-petroleum-based acetone production process would reduce the dramatic volatility in the acetone market and provide a hedge against the impact of alternative production processes. 


\section{LanzaTech Business Model}

As a technology licensor, LanzaTech's business model is predicated on engaging with multiple customers to deploy its technology in parallel. The licensing business model allows the technology to be rolled out more quickly by leveraging the capital resources of multiple parties instead of relying on LanzaTech's capital assets. This model has been successfully used in many industries, including by other process technology companies, such as Honeywell UOP. LanzaTech has also proven this model for its process with the successful commercialization of its gas fermentation technology to produce ethanol in China. There are four additional announced commercial facilities in various stages of design and construction, all also employing the technology licensing business model.

\subsection{Plans for Future Collaboration}

Team scientists at both ORNL and LanzaTech consider their collaboration in the DOEBETO-funded project a huge success that leveraged the unique capabilities of both partners, and, as a result, have sought additional opportunities to continue the collaboration. The microbial genomic, proteomic, and metabolomic resources generated in the project laid the foundation for a succesfully-funded US DOE Office of Biological and Environmental Research (OBER) Biodesign proposal titled "Establishing a clostridia foundry for biosystems design by integrating computational modeling, systems-level analyses, and cell-free engineering technologies" that has led to the establishment of the Clostridium Foundry for Biosystems Design "cBioFab" project that is led by Michael Jewett at Northwestern University (NU) and is a collaboration between NU, LanzaTech, and ORNL. The purpose of the project is to take a systems biology approach to rapidly prototype new gene and pathway variants to develop a novel class of acetogenic clostridia and produce high levels of biofuels and bioproducts from lignocellulosic biomassderived syngas. Novel rapid cell-free engineering technologies are applied in conjunction with genome-wide design and editing, and systems biology studies to develop clostridia as emerging fermentative platform organisms. Again, the genomic resources generated in the DOE-BETOfunded project are critical elements in the follow-on project that will allow the continued collaboration of the partner institutions.

\subsection{Conclusions}

The LanzaTech-led team successfully demonstrated that acetone production via conversion of biomass syngas fermentation with engineered acetogenic bacteria is an environmentally friendly and commercially viable process. We have demonstrated continuous acetone production with stability, rates and yields comparable to the commercial target and in a scalable system. Specific findings from the project include:

Technical achievements and findings:

Acetogenic clostridia bacteria are genetically tractable. To our knowledge this project comprises the largest genetic library and largest number of modifications made in a Clostridium strain to date. A library of several hundred combinatorial strains and nine different genetically modified $C$. autoethanogenum chassis strains that comprise multiple modifications have been generated. 
Expanded pool of acetone biosynthetic genes/enzymes. A total of 200 genomes mined from the industrial $\mathrm{ABE}$ fermentation strain collection were sequenced at the Joint Genome Institute (JGI; A DOE user facility) in a separate effort. Unique ABE acetone pathway genes identified: 29 (thiolase), 9 (CoA transferase subunit A), 19 (CoA transferase subunit B) and 15 (decarboxylase) from the genomes sequenced. Unique synthetic pathway genes identified: 14 (Ptb) and 13 (Buk).

The novel synthetic pathway offers significant advantages. Genome scale modeling (GEM) work confirmed that the synthetic pathway (Ptb-Buk) may offer 3.5x higher yields compared to the standard $\mathrm{ABE}$ fermentation pathway by decoupling acetone production from the production of native fermentation products. Ptb-Buk variants were identified with $\geq 50 \%$ higher selectivity towards acetoacetyl-CoA than acetyl-CoA.

Continuous, scalable process developed. Omics experiments revealed that acetone production instability observed at the start of the project was caused by decreasing expression levels of acetone pathway genes. This led to the construction of an integrated strain which was able to produce acetone and ethanol for over a 3-week period. Process optimizations including, but not limited to, reactor configurations, media composition, and feeding rates led to further improvements in acetone and ethanol co-production and co-selectivity. The LanzaTech acetone process is a scalable process based on the excellent correlation between lab-scale and pilot-scale runs under similar conditions.

Exceeded reported acetone productivity and selectivity of all other biological process to date ${ }^{7-}$ 10.

Technoeconomic and life cycle analyses of the biomass to acetone and ethanol co-production showed that:

The fully developed process provides a cost competitive route to acetone from sustainable resources and enables low-cost biofuels at or below DOE's $\$ 3 /$ gge target.

At the start of the project, the cash cost of production (CCOP) was $>3 \mathrm{X}$ higher than our commercial target. By the end of the project, with each set of strain improvements and process optimizations, the CCOP was within $6 \%$ of our target.

The LanzaTech acetone process provided over $180 \%$ GHG emission reduction (from cradleto-gate) compared to the petrochemical (cumene) process in chemical applications.

The LanzaTech ethanol co-product process provided over $90 \%$ GHG emission reduction (from cradle-to-grave) over fossil gasoline. This would qualify LanzaTech ethanol as a cellulosic biofuel under the Renewable Fuel Standard.

\section{References}

1. Yan, Y. et al. Large-Scale, Three-Dimensional, Free-Standing and Mesoporous Metal Oxide Networks for High-Performance Photocatalysis. Sci. Rep. 3, 4-10 (2013).

2. Anbarasan, P. et al. Integration of chemical catalysis with extractive fermentation to produce fuels. Nature 491, 235 (2012).

3. van Leeuwen, B. N. M., van der Wulp, A. M., Duijnstee, I., van Maris, A. J. A. \& Straathof, A. J. J. Fermentative production of isobutene. Appl. Microbiol. Biotechnol. 93, 1377-1387 (2012).

4. Green chemistry. Available at: http://www.essentialchemicalindustry.org/processes/greenchemistry.html.

5. Jones, D. T. \& Woods, D. R. Acetone-butanol fermentation revisited. Microbiol. Rev. 50, 
484-524 (1986).

6. Köpke, M. \& Dürre, P. Biochemical production of biobutanol. Handb. Biofuels Prod. Process. Technol. 221-257 (2010). doi:10.1533/9780857090492.2.221

7. Luo, H. et al. Effectively enhancing acetone concentration and acetone/butanol ratio in $\mathrm{ABE}$ fermentation by a glucose/acetate co-substrate system incorporating with glucose limitation and C. acetobutylicum/S. cerevisiae co-culturing. Biochem. Eng. J. 118, 132142 (2017).

8. Banerjee, A., Leang, C., Ueki, T., Nevin, K. P. \& Lovley, D. R. A Lactose-Inducible System for Metabolic Engineering of Clostridium ljungdahlii. Appl. Environ. Microbiol. 80, (2014).

9. Hoffmeister, S. et al. Acetone production with metabolically engineered strains of Acetobacterium woodii. Metab. Eng. 36, 37-47 (2016).

10. May, A. et al. A modified pathway for the production of acetone in Escherichia coli. Metab. Eng. 15, 218-225 (2013).

11. O’Brien, E. J., Monk, J. M. \& Palsson, B. O. Using Genome-scale Models to Predict Biological Capabilities. Cell 161, 971-987 (2015).

12. Marcellin, E. et al. Low carbon fuels and commodity chemicals from waste gases Systematic approach to understand energy metabolism in a model acetogen. Green Chem. 3020-3028 (2016). doi:10.1039/C5GC02708J

13. Köpke, M. et al. Reconstruction of an Acetogenic 2,3-Butanediol Pathway Involving a Novel NADPH-Dependent Primary-Secondary Alcohol Dehydrogenase. Appl. Environ. Microbiol. 80, 3394-403 (2014).

14. Heap, J. T., Pennington, O. J., Cartman, S. T., Carter, G. P. \& Minton, N. P. The ClosTron: a universal gene knock-out system for the genus Clostridium. J. Microbiol. Methods 70, 452-64 (2007).

15. Koepke, M., Al-Sinawi, B., Jensen, R. O. \& Hill, R. E. WO2015085015A1.pdf. (2015).

16. Dong, H., Tao, W., Zhang, Y. \& Li, Y. Development of an anhydrotetracycline-inducible gene expression system for solvent-producing Clostridium acetobutylicum: A useful tool for strain engineering. Metab. Eng. 14, 59-67 (2012).

17. Liew, F. M. et al. Gas Fermentation-A flexible platform for commercial scale production of low-carbon-fuels and chemicals from waste and renewable feedstocks. Frontiers in Microbiology 7, (2016).

18. Trapnell, C. et al. Differential analysis of gene regulation at transcript resolution with RNA-seq. Nat. Biotechnol. 31, 46 (2012).

19. Love, M. I., Huber, W. \& Anders, S. Moderated estimation of fold change and dispersion for RNA-seq data with DESeq2. Genome Biol. 15, 550 (2014).

20. Mock, J. et al. Energy Conservation Associated with Ethanol Formation from $\mathrm{H} 2$ and $\mathrm{CO} 2$ in Clostridium autoethanogenum Involving Electron Bifurcation. J. Bacteriol. 197, 2965-2980 (2015).

21. Marcellin, E. et al. Low carbon fuels and commodity chemicals from waste gases Systematic approach to understand energy metabolism in a model acetogen. Green Chem. (2016). doi:10.1039/C5GC02708J

22. Köpke, M. et al. Reconstruction of an Acetogenic 2,3-Butanediol Pathway Involving a Novel NADPH-Dependent Primary-Secondary Alcohol Dehydrogenase. Appl. Environ. Microbiol. 80, 3394-403 (2014). 
